\title{
Interval estimation via tail functions
}

\author{
Borek Puza ${ }^{1,2}$ and Terence O'Neill ${ }^{1}, 6$ May 2005 \\ ${ }^{1}$ School of Finance and Applied Statistics, Australian National University, \\ ACT 0200, Australia. ${ }^{2}$ Corresponding author: borek.puza@anu.edu.au
}

\begin{abstract}
In this paper we describe a new methodology for constructing confidence intervals. The idea is to specify the tail cutoff areas in terms of a function of the target parameter rather than as constants. This function, called the tail function, can be engineered so as to provide shorter confidence intervals when prior information is available. It can also be used to improve the coverage properties of approximate confidence intervals. We illustrate the methodology by applying it to inference on the normal mean and binomial proportion, and develop measures of the resulting improvements. Guidelines for choosing the optimal tail function in any situation are provided, and the relationship with Bayesian inference is discussed.
\end{abstract}

\section{Key words}

Confidence interval, shorter, prior, tail function, Wilson, Clopper-Pearson, Blaker.

\section{Introduction}

The classical theory of two-sided confidence intervals (CI's) (Stuart et al., 1999) involves inverting a probability statement regarding a pivotal quantity, $Y$, so as to yield two statistics which 'straddle' the quantity of interest, $\theta$. This theory requires the specification of a confidence level, $1-\alpha$ (e.g. 0.95), which 'cuts off' an area of $\alpha / 2$ from either tail of $Y$ 's distribution. In the statistical literature the tail areas have always been taken as constant. The new method involves specifying these areas in terms of a function of $\theta$, called the tail function, and then proceeding to invert the probability statement to create a different $\mathrm{CI}$ for $\theta$. The main advantage of this approach is that it can be used to produce shorter CI's when prior information is available. It also provides a mechanism for improving the coverage properties of approximate CI's for parameters of discrete distributions. 
The classical theory of CI's is reviewed in Section 2 and generalised in Section 3 by way of tail functions. Section 4 shows how these functions can be used in conjunction with prior information to produce shorter CI's for a normal mean when the normal variance is known. We define measures of the improvements which may be achieved, such as the maximum reduction in interval width. We also develop guidelines for selecting the optimal tail function in any situation, and make comparisons with the Bayesian approach. Section 5 then deals with the case of unknown variance, aided by Monte Carlo methods. Section 6 shows how tail functions can be used to produce attractive variants of the 'standard', Wilson and Clopper-Pearson intervals for a binomial proportion. Section 7 concludes with some general discussion and advice.

\section{The classical theory of two-sided confidence intervals}

Consider a scalar probability distribution which is dependent on a single unknown constant parameter, $\theta$, and suppose that we are given a random sample of $n$ observations, $X_{1}, \ldots, X_{n}$, from that distribution, with realised or possible values denoted $x_{1}, \ldots, x_{n}$. Let $X$ and $x$ denote the vectors $\left(X_{1}, \ldots, X_{n}\right)$ and $\left(x_{1}, \ldots, x_{n}\right)$, respectively. Next let $Y=g(X, \theta)$ be a scalar function of $X$ and $\theta$ with realised or possible value denoted $y=g(x, \theta)$, and suppose that the cumulative distribution function (cdf) of $Y$, denoted $F_{Y}(y)$, is continuous and does not depend on $\theta$. Then $F_{Y}(Y)$ has the standard uniform distribution, and we call $Y$ a pivotal quantity. Next choose the confidence level, $1-\alpha$, where $\alpha \in(0,1)$. Then it is true, for any value which $\theta$ may take, that

$$
1-\alpha=P\left(\alpha / 2<F_{Y}(g(X, \theta))<1-\alpha / 2\right) .
$$

The classical theory of CI's involves manipulating (1) so as to produce the statement

$$
1-\alpha=P(L(X)<\theta<U(X)),
$$

where $L$ and $U$ are two functions of $X$ which do not depend on $\theta$. The $1-\alpha$ confidence interval (CI) for $\theta$ is then defined as $(l, u)$, where $l$ and $u$ are the solutions in $\theta$ of the following equations, respectively:

$$
\begin{aligned}
& F_{Y}(g(x, \theta))=1-\alpha / 2 \\
& F_{Y}(g(x, \theta))=\alpha / 2 .
\end{aligned}
$$


In many cases these equations can be solved analytically or at least easily using standard statistical software. However, in general they may require an iterative search procedure such as the Newton-Raphson algorithm. Explicitly, to solve (4) we choose a suitable starting value $\theta_{0}$ and repeatedly calculate $\theta_{j+1}=\theta_{j}-\psi\left(\theta_{j}\right) / \psi^{\prime}\left(\theta_{j}\right)$ until convergence, where:

$$
\begin{aligned}
& \psi(\theta)=F_{Y}(g(x, \theta))-\alpha / 2 \\
& \psi^{\prime}(\theta)=\frac{\partial}{\partial \theta} F_{Y}(g(x, \theta))=f_{Y}(g(x, \theta)) \frac{\partial}{\partial \theta} g(x, \theta) .
\end{aligned}
$$

In (6), $f_{Y}(y)=F_{Y}^{\prime}(y)$ denotes the probability density function (pdf) of $Y$. Equation (3) can be solved in the same way as (4) but with $\alpha / 2$ in (5) replaced by $1-\alpha / 2$.

\section{A generalisation of the classical theory via tail functions}

Consider $\tau(\theta)$, a function of $\theta$ which is nondecreasing and has a range in the interval from 0 to 1 . Then just as (1) is true for all $\theta$, so also

$$
1-\alpha=P\left(\alpha \tau(\theta)<F_{Y}(g(X, \theta))<1-\alpha+\alpha \tau(\theta)\right) .
$$

We now recognise (1) as being a special case of (7), namely where $\tau(\theta)=1 / 2$ (a constant). Suppose that it is possible to invert (7) so as to produce a statement of the form (2). Then a $1-\alpha$ CI's for $\theta$ is $(l, u)$, where $l$ and $u$ are the solutions in $\theta$ of the following equations, respectively:

$$
\begin{aligned}
& F_{Y}(g(x, \theta))=1-\alpha+\alpha \tau(\theta) \\
& F_{Y}(g(x, \theta))=\alpha \tau(\theta) .
\end{aligned}
$$

Equations (8) and (9) are solvable via the Newton-Raphson algorithm in the same way as (3) and (4). Explicitly, to solve (9) we choose a suitable starting value $\theta_{0}$ and repeatedly calculate $\theta_{j+1}=\theta_{j}-\psi\left(\theta_{j}\right) / \psi^{\prime}\left(\theta_{j}\right)$ until convergence, where:

$$
\begin{aligned}
& \psi(\theta)=F_{Y}(g(x, \theta))-\alpha \tau(\theta) \\
& \psi^{\prime}(\theta)=f_{Y}(g(x, \theta)) \frac{\partial}{\partial \theta} g(x, \theta)-\alpha \tau^{\prime}(\theta), \quad \tau^{\prime}(\theta)=\frac{\partial}{\partial \theta} \tau(\theta) .
\end{aligned}
$$

Equation (8) can be solved in the same way as (9) but with $\alpha \tau(\theta)$ in (10) replaced by $1-\alpha+\alpha \tau(\theta)$, and with (11) unchanged. We call $\tau(\theta)$ the tail function. 


\section{Inference on a normal mean with known variance}

To illustrate the new technique, suppose that we have a random sample $X_{1}, \ldots, X_{n}$ from the normal distribution with unknown mean $\mu$ and known variance $\sigma^{2}$. We wish to construct a $1-\alpha \mathrm{CI}$ for $\mu$. The classical approach involves using the result

$$
Y=\frac{\bar{X}-\mu}{\sigma / \sqrt{n}} \sim N(0,1), \quad \text { where } \bar{X}=\frac{1}{n} \sum_{i=1}^{n} X_{i} \text { is the sample mean, }
$$

and taking the tail function as $\tau(\mu)=1 / 2,-\infty<\mu<\infty$ (constant). The resulting CI is $\left(\bar{x} \pm z_{\alpha / 2} \sigma / \sqrt{n}\right)$, where $\bar{x}=\left(x_{1}+\ldots+x_{n}\right) / n$ and $z_{p}$ denotes the upper $p$-quantile of the standard normal distribution.

Let us now consider a different tail function, one of the form

$$
\tau(\mu)=\delta+(1-2 \delta) \Phi\left(\frac{\mu-\eta}{\lambda}\right)
$$

where $\eta \in \Re, \lambda \geq 0,0 \leq \delta \leq 1 / 2$, and $\Phi(z)$ denotes the standard normal cdf. This tail function is nondecreasing, with values $\delta$ at $-\infty, 1 / 2$ at $\eta$, and $1-\delta$ at $+\infty$. It is also continuous if $\lambda>0$, in which case its slope is given by

$$
\tau^{\prime}(\mu)=\frac{\partial}{\partial \mu} \tau(\mu)=\left(\frac{1-2 \delta}{\lambda}\right) \phi\left(\frac{\mu-\eta}{\lambda}\right)
$$

where $\phi(t)$ denotes the standard normal pdf. Several examples of $\tau(\mu)$ are shown in Figure 1. Note that $\delta$ determines the minimum deviation of $\tau(\mu)$ from 0 or $1, \eta$ is the value of $\mu$ at which $\tau(\mu)$ equals $1 / 2$, and $\lambda$ is inversely proportionate to the slope of $\tau(\mu)$, whose maximum value is $\tau^{\prime}(\eta)=(1-2 \delta) /(\lambda \sqrt{2 \pi})$.

We will refer to the tail function (12) and associated CI's as Gaussian with parameters $\eta, \delta$ and $\lambda$, although the terms modified, alternative and new will often be used instead. Observe that $\tau(\mu)$ reduces to $1 / 2$ if $\delta=1 / 2$ or $\lambda=\infty$. In that case we refer to $\tau(\mu)$ and associated CI's as ordinary or classical. 
Figure 1 Several Gaussian tail functions

$\tau(\mu)$

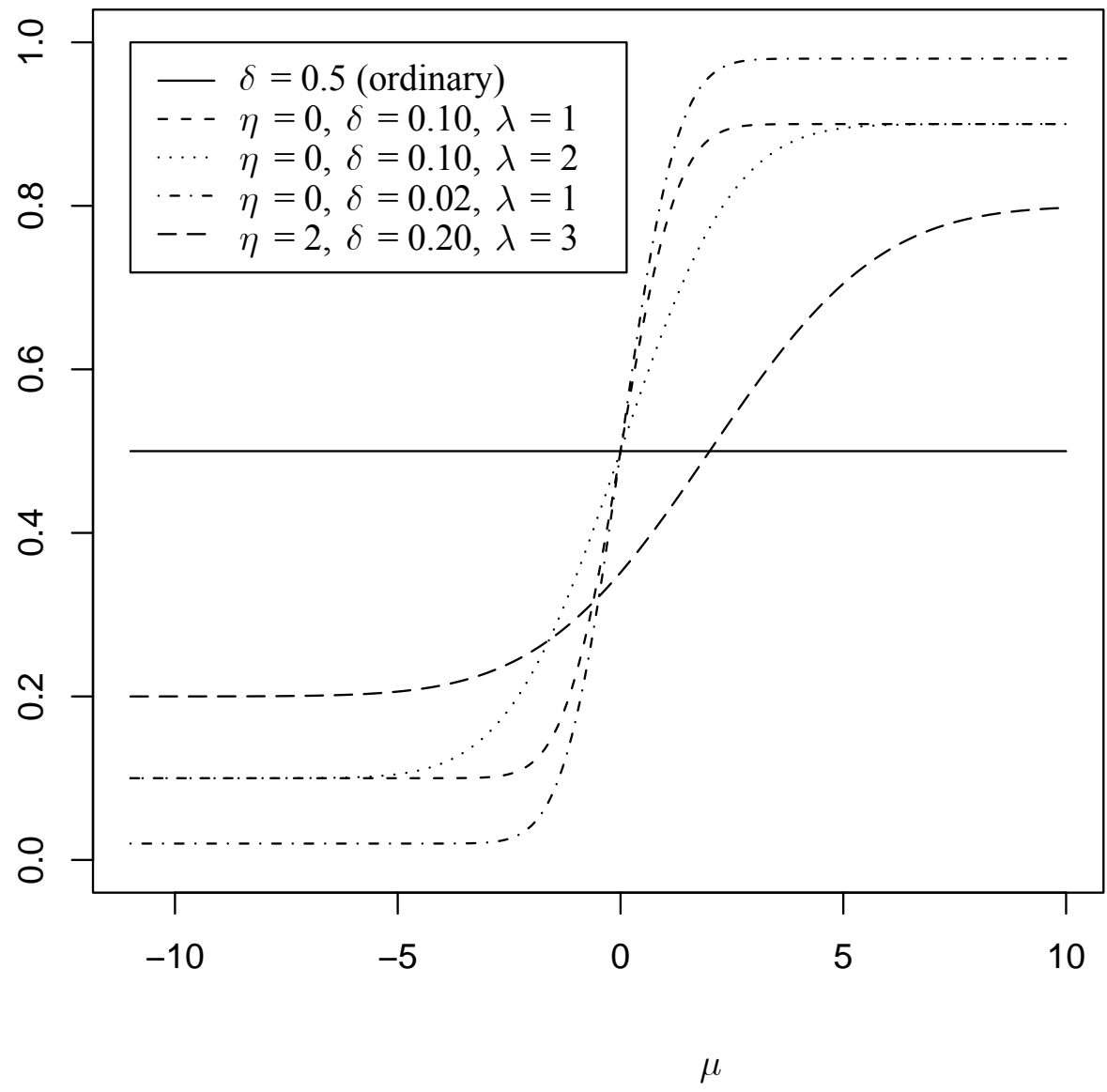

To find the lower bound of a Gaussian $1-\alpha \mathrm{CI}$, we choose a suitable starting value $\mu_{0}$ and repeatedly calculate $\mu_{j+1}=\mu_{j}-\psi\left(\mu_{j}\right) / \psi^{\prime}\left(\mu_{j}\right)$ until convergence, where:

$$
\begin{aligned}
& \psi(\mu)=\Phi\left(\frac{\bar{x}-\mu}{\sigma / \sqrt{n}}\right)-\alpha \tau(\mu) \\
& \psi^{\prime}(\mu)=\phi\left(\frac{\bar{x}-\mu}{\sigma / \sqrt{n}}\right)\left(\frac{-1}{\sigma / \sqrt{n}}\right)-\alpha \tau^{\prime}(\mu) .
\end{aligned}
$$

The upper bound can be found in the same way but with $\alpha \tau(\mu)$ in (14) replaced by $1-\alpha+\alpha \tau(\mu)$, and with (15) unchanged. S-PLUS code (Venables and Ripley, 1999) and $R$ code (Venables and Smith, 2004) for calculating these bounds and those of all other CI's in this paper can be obtained from the authors upon request. 


\section{Example 1}

Suppose that $n=4, \bar{x}=55$ and $\sigma=17$. Then the classical 95\% CI for $\mu$ is $(55 \pm 1.96(17) / \sqrt{4})=(38.3,71.7)$. Implementing the above Newton-Raphson algorithm with starting values equal to the classical bounds, and with $\eta=50, \delta=1 / 8$ and $\lambda=10$, we find that the new CI is $(39.9,69.6)$. Observe that the lengths of these two intervals are 33.3 and 29.7, respectively. Thus the alternative tail function has resulted in a width reduction of about $11 \%$.

To see what is going on, let us plot the classical and new confidence limits for all values of $\bar{x}$ from 0 to 100 . Figure 2 illustrates, with the bounds of the two CI's in Example 1 shown as points (see Note 1). We see that the alternative tail function creates a 'distortion' of the classical bounds, so that when $\bar{x}$ is near $\eta=50$, the new $\mathrm{CI}$ is narrower. This effect is compensated for by the new CI being somewhat wider when $\bar{x}$ is far from $\eta$. We find that this pattern is symmetric about $\eta$. Thus the alternative CI has a minimum width at $\bar{x}=\eta$, and at $\bar{x}=45$ its width is the same as at $\bar{x}=55$, i.e. 29.7 .

With these observations we have established the significance of the parameter $\eta$. It should be specified as a value of $\mu$ which is deemed very likely a priori, for then $\bar{X}$ will likely be close to $\eta$ and hence the resulting CI will likely be narrower than the ordinary CI. This idea will be made more precise later on. 
Figure 2 Two sets of $95 \%$ confidence bounds when $n=4, \sigma=17, \eta=\mathbf{5 0}$, $\delta=\mathbf{1} / \mathbf{8}$ and $\lambda=\mathbf{1 0}$

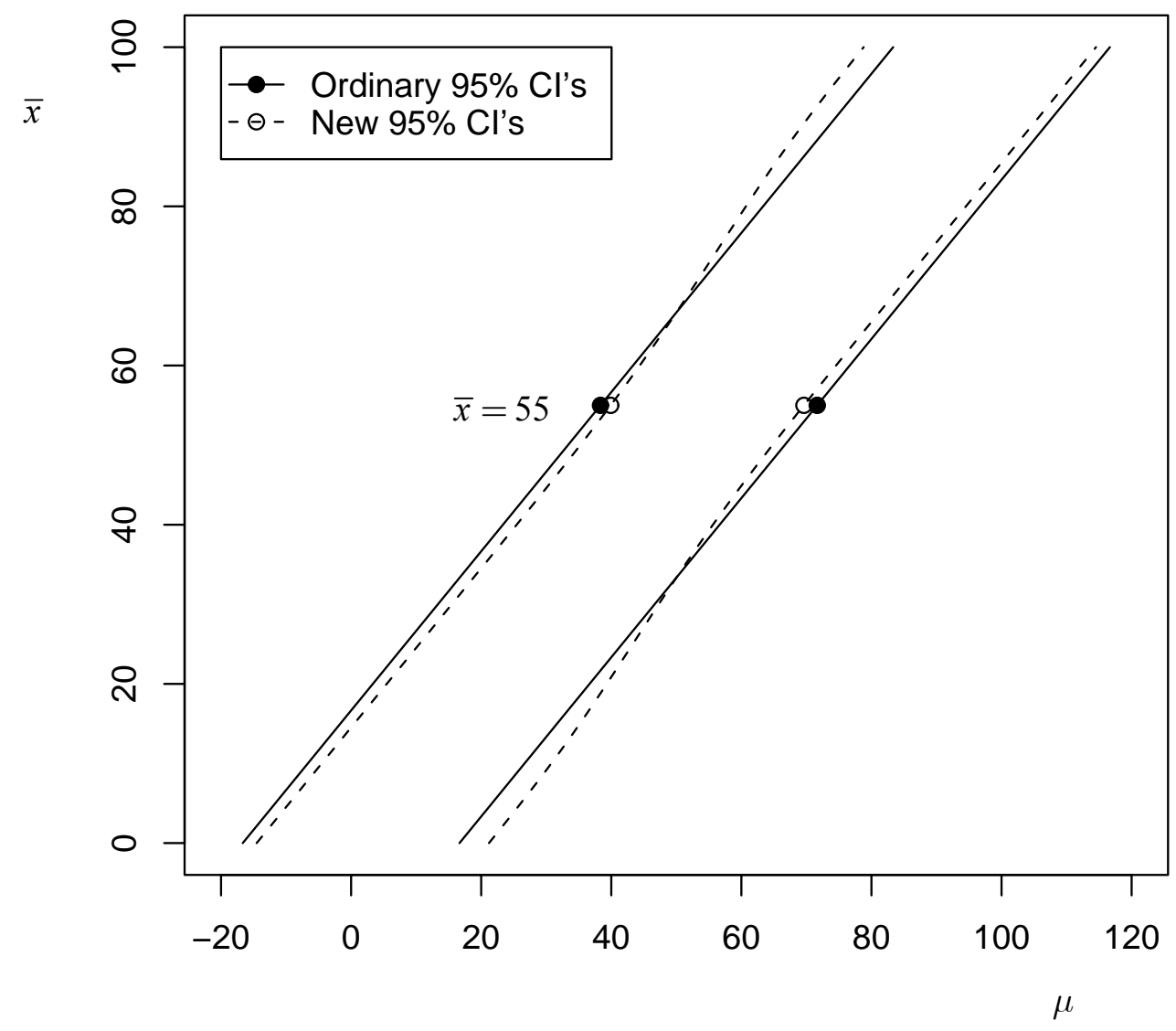

\section{The significance of $\delta$ and $\lambda$}

To gain an understanding of the parameters $\delta$ and $\lambda$, it is useful to examine some more examples. Figure 3 shows, for $\eta=0$ and $n=\sigma=1$, the $95 \%$ confidence bounds implied by the first four tail functions in Figure 1 (including the 'ordinary' one). We see that $\delta$ determines the minimum and maximum possible lengths of the $\mathrm{CI}$, and $\lambda$ influences the rate at which those extrema are approached as $\bar{x}$ tends to $\eta$ or $\pm \infty$. Specifying a small value of $\delta$ results in a relatively short interval if $\bar{x}$ is close to $\eta$ and a wide one otherwise. Specifying a large value of $\lambda$ has the effect of evening out the disturbance due to $\delta$, whilst not altering the crossover points, given generally by $(\mu, \bar{x})=\left(\eta, \eta \pm z_{\alpha / 2} \sigma / \sqrt{n}\right)$. Examples of these points are $(0, \pm 1.96)$ in Figure 3 , and $(50,50 \pm 1.96(17) / \sqrt{4})=(50,33.3)$ and $(50,66.7)$ in Figure 2. 
Figure 3 Several sets of $95 \%$ confidence bounds when $n=\sigma=1$ and $\eta=0$

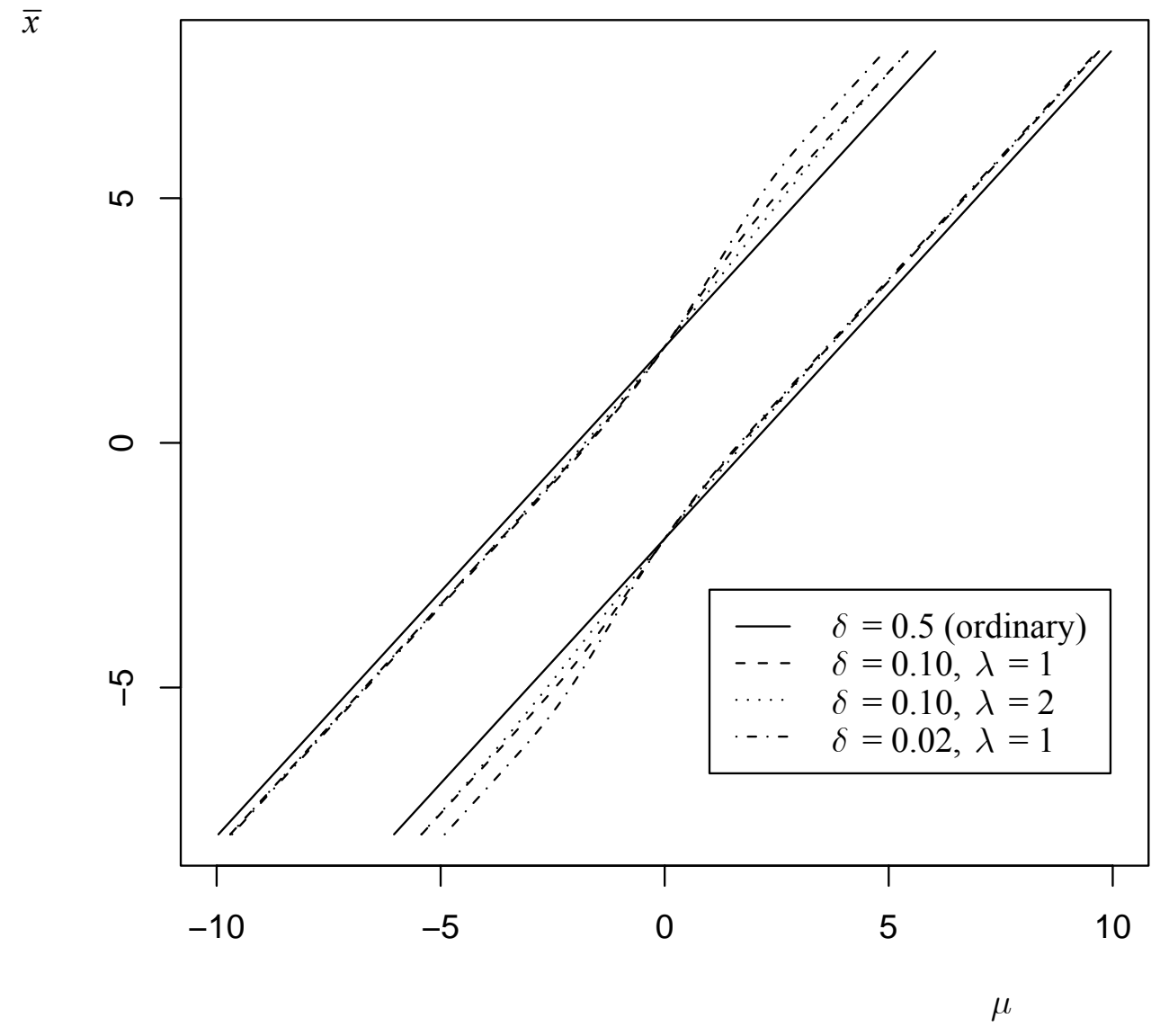

The PDL and EPDL

A convenient way to quantify the length (or width) of a modified CI is in terms of the proportion by which it is shorter than the corresponding ordinary CI. This leads us to define the proportional decrease in length $(P D L)$ as

$$
D_{\delta, \lambda}(\bar{x})=\frac{W-W_{\delta, \lambda}(\bar{x})}{W}
$$

where $W_{\delta, \lambda}(\bar{x})=u-l$ is the length of the modified CI and $W=2 z_{\alpha / 2} \sigma / \sqrt{n}$ is the length of the ordinary CI. 
Another useful quantity is the expected proportional decrease in length (EPDL), which may be defined as

$$
e_{\delta, \lambda}(\mu)=E\left\{D_{\delta, \lambda}(\bar{X}) \mid \mu\right\}=\int_{-\infty}^{\infty} D_{\delta, \lambda}(\bar{x}) f(\bar{x} \mid \mu) d \bar{x}
$$

where $f(\bar{x} \mid \mu)=\phi((\bar{x}-\mu) \sqrt{n} / \sigma) \sqrt{n} / \sigma$, since $(\bar{X} \mid \mu) \sim N\left(\mu, \sigma^{2} / n\right)$.

Figure 4 shows both the PDL and EPDL for each of the three new CI's in Figure 3. The first of these two functions shows clearly the values of $\bar{x}$ for which the CI is narrower than the ordinary CI (a neighbourhood around $\eta=0$ ). In contrast, the latter function shows the values of $\mu$ for which the CI is expected to be narrower (again near $\eta$ ). Both the PDL and EPDL are symmetric about and have a maximum at $\eta$.

Figure 4 Examples of the PDL and EPDL when $n=\sigma=1$ and $\eta=0$

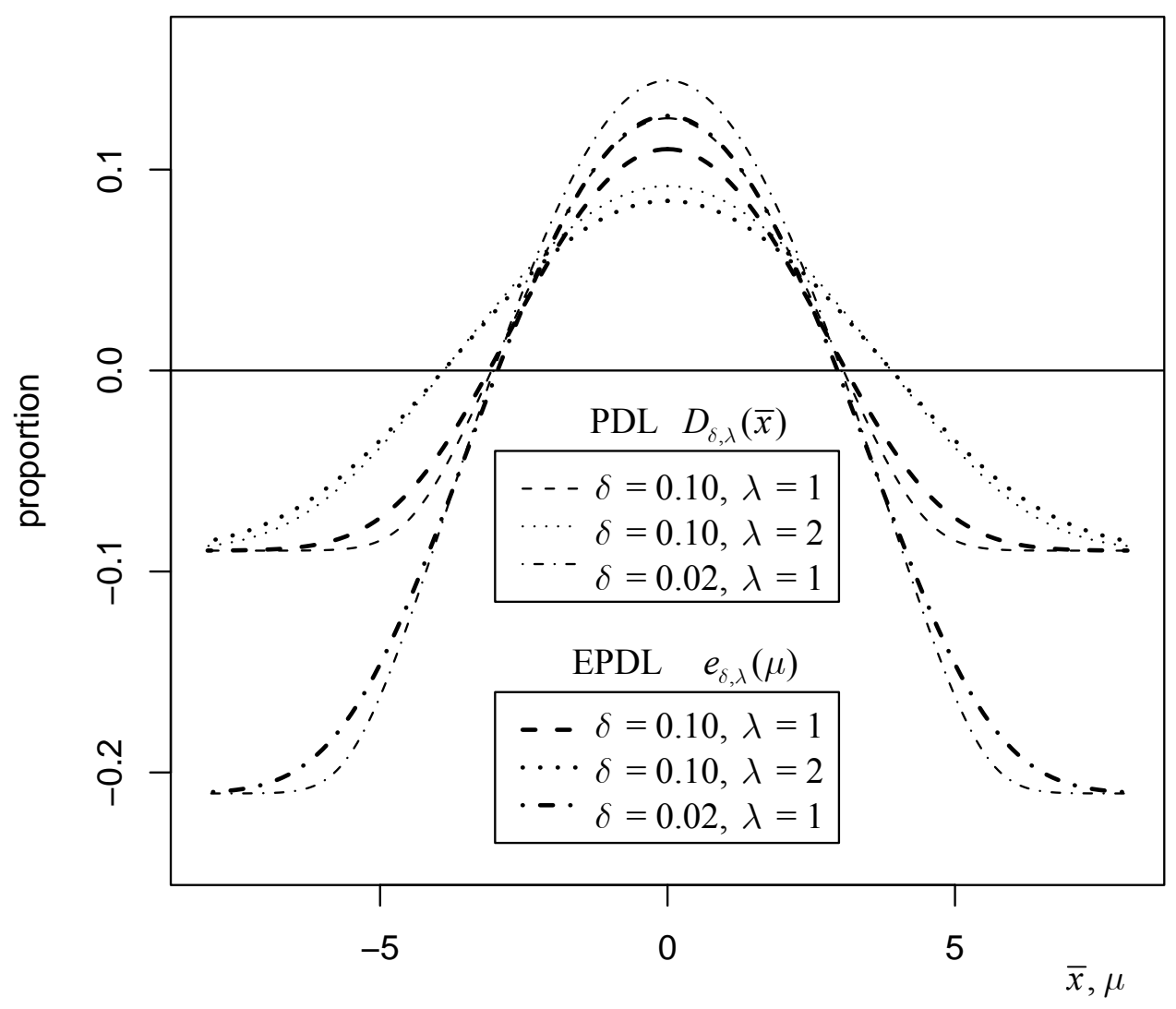




\section{The MPIL}

It is also useful to be able to quantify the extent by which a CI in the new class may be worse than the ordinary CI. It can be shown that the maximum width of a modified CI is $\tilde{W}=W_{\delta, \lambda}(\infty)=\left(z_{\alpha \delta}+z_{\alpha(1-\delta)}\right) \sigma / \sqrt{n}$. Accordingly, we define the maximum proportional increase in length (MPIL) as

$$
I_{\delta}=\frac{\tilde{W}-W}{W}=\frac{z_{\alpha \delta}+z_{\alpha(1-\delta)}}{2 z_{\alpha / 2}}-1 .
$$

For example if $\alpha=0.05$ then $I_{0.1}=0.0896$. Observe that the MPIL does not depend on $\lambda$. Also, it is strictly increasing and converges to infinity as $\delta$ tends to zero.

It is a fact that decreasing $\delta$ always has the effect of increasing the maximum possible decrease in length, namely at $\bar{x}=\eta$ (see Figures 3 and 4). This suggests a useful rule. Suppose we want to be sure that the new CI does not exceed the old CI in length by more than $100 I \%$. Then we should specify $\delta$ as the value for which $I_{\delta}=I$.

For example, if $I=0.1$ and $\alpha=0.05$, then we find using the Newton-Raphson algorithm that $\delta=0.0871$ (see Note 2). Figure 5 illustrates the relationship between $\delta$ and the MPIL for various values of $\alpha$, with the two points being at $\left(I_{\delta}, \delta\right)=(0.0896,0.1)$ and $(0.1,0.0871)$. 
Figure 5 Relationship between $\delta$ and the MPIL

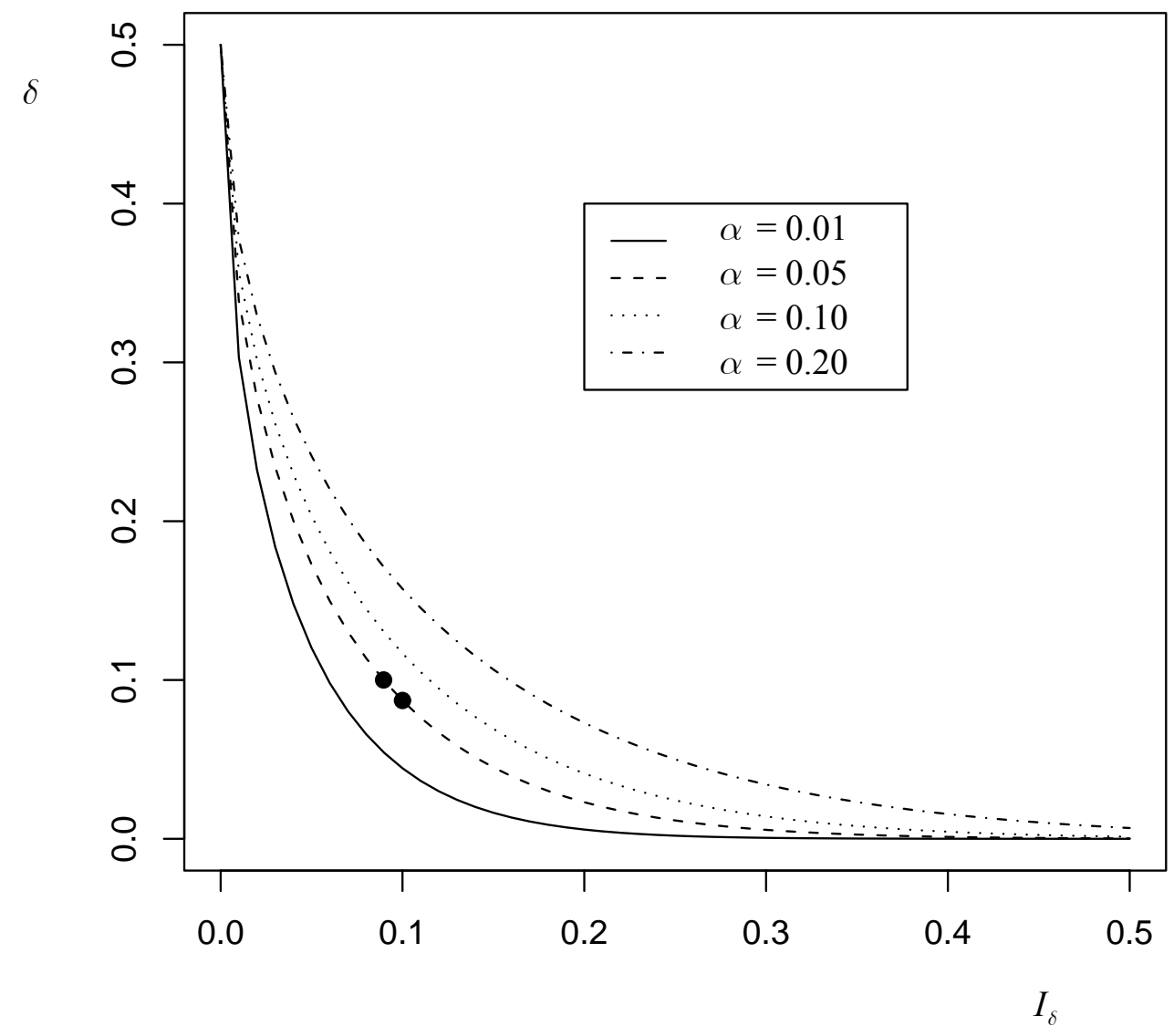

The MPDL, GMPDL and AGMPDL

Recall that a modified CI is always narrowest at $\bar{x}=\eta$. Accordingly we define the maximum proportional decrease in length $(M P D L)$ as $M_{\delta, \lambda}=D_{\delta, \lambda}(\eta)$. We find that for a given $\delta$, this quantity is a nonincreasing function of $\lambda$ and so has a maximum at $\lambda=0$. Accordingly, we define the greatest maximum proportional decrease in length $(G M P D L)$ as $G_{\delta}=M_{\delta, 0}$. We then find that $G_{\delta}$ is a decreasing function of $\delta$. Accordingly, we define the absolute greatest maximum proportional decrease in length $(A G M P D L)$ as $A=G_{0}$. The following are formulae for all these quantities:

$$
\begin{array}{ll}
D_{\delta, \lambda}(\bar{x})=1-W_{\delta, \lambda}(\bar{x}) / W & (\mathrm{PDL}) \\
M_{\delta, \lambda}=D_{\delta, \lambda}(\eta)=1-W_{\delta, \lambda}(\eta) / W & (\mathrm{MPDL}) \\
G_{\delta}=M_{\delta, 0}=1-z_{\alpha(1-\delta)} / z_{\alpha / 2} & (\mathrm{GMPDL}) \\
A=G_{0}=1-z_{\alpha} / z_{\alpha / 2} & \text { (AGMPDL). }
\end{array}
$$


Figure 4 shows three values of the MPDL when $\alpha=0.05$ and $\sigma=n=1$, namely the heights of the three thin lines at $\bar{x}=\eta=0$. These values are $M_{0.1,1}=0.1255$, $M_{0.1,2}=0.0918$ and $M_{0.02,1}=0.1443$. Thus, for example, if $\delta=0.1$ and $\lambda=2$, the improvement in interval length is limited to $9.2 \%$.

To see the significance of the GMPDL, recall that a MPIL of $10 \%$ implies $\delta=0.0871$. The associated GMPDL is $G_{0.0871}=1-z_{0.05(1-0.0871)} / z_{0.05 / 2}=0.138$. This means that if we want to be sure that the new $95 \% \mathrm{CI}$ will be no more than $10 \%$ wider than the ordinary $95 \% \mathrm{CI}$, we must also accept that it will be no more than $13.8 \%$ narrower, with that limit achievable only if $\lambda=0$.

The AGMPDL provides an absolute upper bound on the improvement to a $1-\alpha \mathrm{CI}$ which can be achieved. For example, if $\alpha=0.05$ then $A=1-1.645 / 1.96=0.161$. Thus a modified $95 \%$ CI can be no more than $16.1 \%$ narrower than an ordinary $95 \%$ $\mathrm{CI}$, regardless of the values of $\bar{x}, \sigma, n, \eta, \delta$ and $\lambda$. Some other examples of the AGMPDL are $0.097,0.221,0.343$ and 1.000 when $\alpha=0.01,0.10,0.20$ and 0.50 , respectively. From this we see that modification via tail functions is potentially most effective if an interval with small confidence level is required.

Figure 6 provides further illustrations of the MPDL, GMPDL and AGMPDL, with some of the above numbers shown as points. The height of each line at the origin represents a GMPDL, and the height of the highest line at the origin is the AGMPDL for the case $\alpha=0.05$. For further discussion of Figure 6 see Note 3 . 
Figure 6 Examples of the MPDL, GMPDL and AGMPDL

when $n=\sigma=1$ and $\alpha=\mathbf{0 . 0 5}$

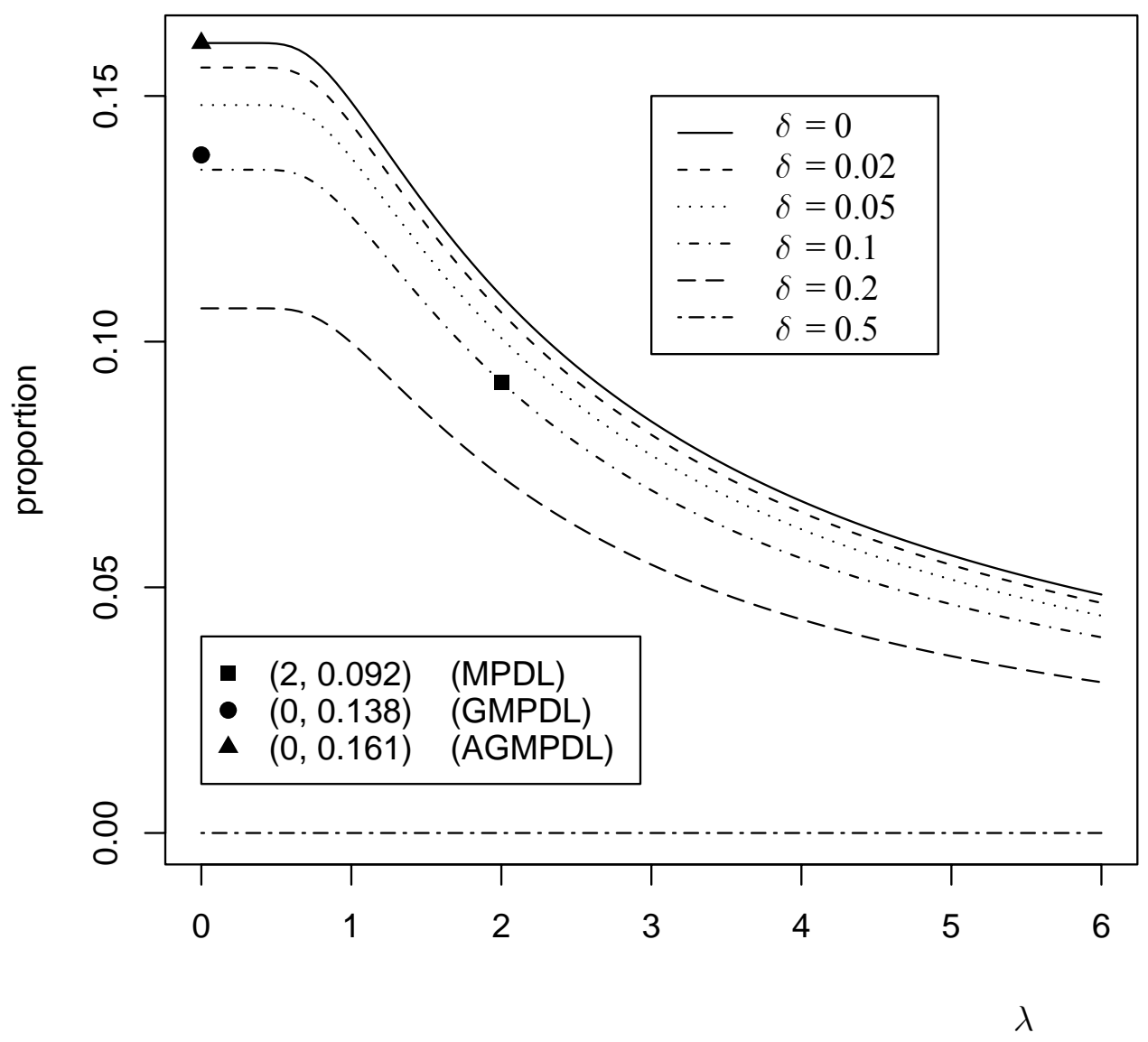

The case of $\lambda=0$

In the limiting case $\lambda=0$, the new interval can be written tractably, with no need for the Newton-Raphson algorithm. With $\kappa=\sigma / \sqrt{n}$, its bounds are then:

$$
\begin{array}{lll}
\left(\bar{x}-z_{\alpha \delta} \kappa,\right. & \left.\bar{x}+z_{\alpha(1-\delta)} \kappa\right), & \bar{x}>\eta+\kappa z_{\alpha \delta} \\
(\eta, & \left.\bar{x}+z_{\alpha(1-\delta)} \kappa\right), & \eta+z_{\alpha(1-\delta)} \kappa \leq \bar{x}<\eta+z_{\alpha \delta} \kappa \\
\left(\bar{x}-z_{\alpha(1-\delta)} \kappa,\right. & \left.\bar{x}+z_{\alpha(1-\delta)} \kappa\right), & \eta-z_{\alpha(1-\delta)} \kappa \leq \bar{x} \leq \eta+z_{\alpha(1-\delta)} \kappa \\
\left(\bar{x}-z_{\alpha(1-\delta)} \kappa,\right. & \eta), & \eta-z_{\alpha \delta} \kappa \leq \bar{x}<\eta-z_{\alpha(1-\delta)} \kappa \\
\left(\bar{x}-z_{\alpha(1-\delta)} \kappa,\right. & \left.\bar{x}+z_{\alpha \delta} \kappa\right), & \bar{x}<\eta-z_{\alpha \delta} \kappa
\end{array}
$$


Figure 7 shows $95 \%$ CI's corresponding to various values of $\delta$ when $\eta=\lambda=0$ and $\sigma=n=1$. The case $\lambda=0$ is important because it implies the maximum possible improvement at $\bar{x}=\eta$ for a given $\delta$. It thereby provides a convenient benchmark against which other values of $\lambda$ may be assessed. Note that when $\lambda=0$ the tail function (12) is discontinuous and consists of a single step up of $1-2 \delta$ (from $\delta$ to $1-\delta)$ at $\mu=\eta$. We will refer to this tail function and associated CI's such as those in Figure 7 as maximal (or maximal Gaussian).

Figure 7 Several maximal 95\% CI's $(\lambda=0)$ when $\boldsymbol{n}=\sigma=\mathbf{1}$ and $\eta=\mathbf{0}$

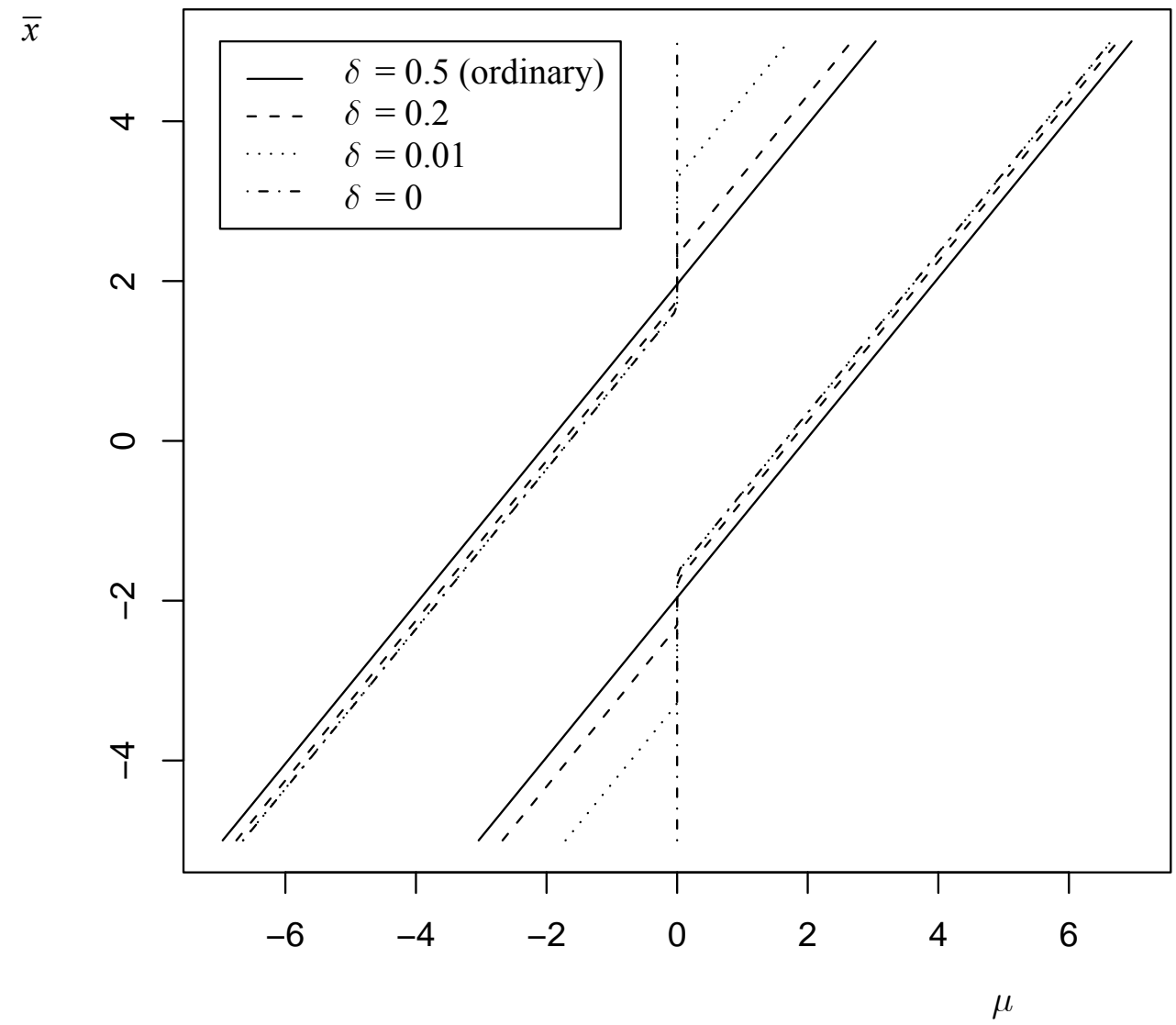




\section{The PEPDL}

In order to choose useful values of $\eta, \delta$ and $\lambda$, we must have in mind some prior distribution for $\mu$. Suppose that this prior is normal with mean $\mu_{0}$ and standard deviation $\sigma_{0}$ (see Note 4). In that case we should take $\eta=\mu_{0}$ and may set $\delta$ at the value implied by the desired MPIL. That leaves only $\lambda$ to be specified. One possibility is to let $\lambda=0$, because then the PDL at (16) will be maximised. However, it may be preferable to take $\lambda$ as the value $\lambda_{0}$ which maximizes the prior expected proportional decrease in length (PEPDL), defined as

$$
p_{\delta}(\lambda)=E D_{\delta, \lambda}(\bar{X})=\int_{-\infty}^{\infty} \int_{-\infty}^{\infty} D_{\delta, \lambda}(\bar{x}) f(\bar{x} \mid \mu) f(\mu) d \bar{x} d \mu
$$

where $f(\mu)=\phi\left(\left(\mu-\mu_{0}\right) / \sigma_{0}\right) / \sigma_{0}$ and $f(\bar{x} \mid \mu)=\phi((\bar{x}-\mu) \sqrt{n} / \sigma) \sqrt{n} / \sigma$. For other ways to write the PEPDL see Note 5.

Calculation of $\lambda_{0}$ requires a search by trial and error. Figure 8 shows the PEPDL at each $\lambda=0,0.01,0.02, \ldots, 1$ for various values of $\sigma_{0}$ when $n=\sigma=1, \delta=0.1, \mu_{0}=0$ and $\alpha=0.05$. For example, if $\sigma_{0}=1$ then the PEPDL is maximized at $\lambda=0.22$, to the nearest 0.01 . Refining our search we find that $\lambda_{0}=0.2233$ and $p_{0.1}\left(\lambda_{0}\right)=0.1053$. We also find that $p_{0.1}(0)=0.1042$. Thus in this case there was not much gain from finding $\lambda_{0}$ (only a $1.0 \%$ decrease in the PEPDL). For inference we may as well take $\lambda$ as zero and use the simple formulae at (20).

For the case $\sigma_{0}=2$, we find that the optimal value of $\lambda$ is 0.9123 , with associated PEPDL 0.0664 . This amounts to an improvement of $14.5 \%$ over 0.0580 , the PEPDL when $\lambda=0$. From this we see that when the prior information is more diffuse, there are greater benefits to be had from finding $\lambda_{0}$. On the other hand, less prior information implies a smaller possible improvement. This is illustrated in Figure 8 where the maxima uniformly decrease as the prior standard deviation $\sigma_{0}$ increases.

Observe that $0.9123 \approx 0.2233 \times 4$. Thus $\lambda_{0}$ at $\sigma_{0}=2$ is roughly $2^{2}$ times $\lambda_{0}$ at $\sigma_{0}=1$. Also, $\lambda_{0}=0$ when $\sigma_{0}=0$. These facts are part of a general pattern which may be written $\lambda_{0} \approx k_{\delta} \sigma_{0}^{2} \sqrt{n} / \sigma$. Three examples of the constant $k_{\delta}$ here are $k_{0.1}=0.22, k_{0.05}=0.27$ and $k_{0.20}=0.16$. Although this formula is only approximate it does provide some guidance to conducting the search for $\lambda_{0}$ in any given situation. 
Figure 8 Examples of the PEPDL and its maximization

when $\alpha=\mathbf{0 . 0 5}, \boldsymbol{n}=\sigma=\mathbf{1}, \eta=\mu_{0}=\mathbf{0}$ and $\delta=\mathbf{0 . 1}$

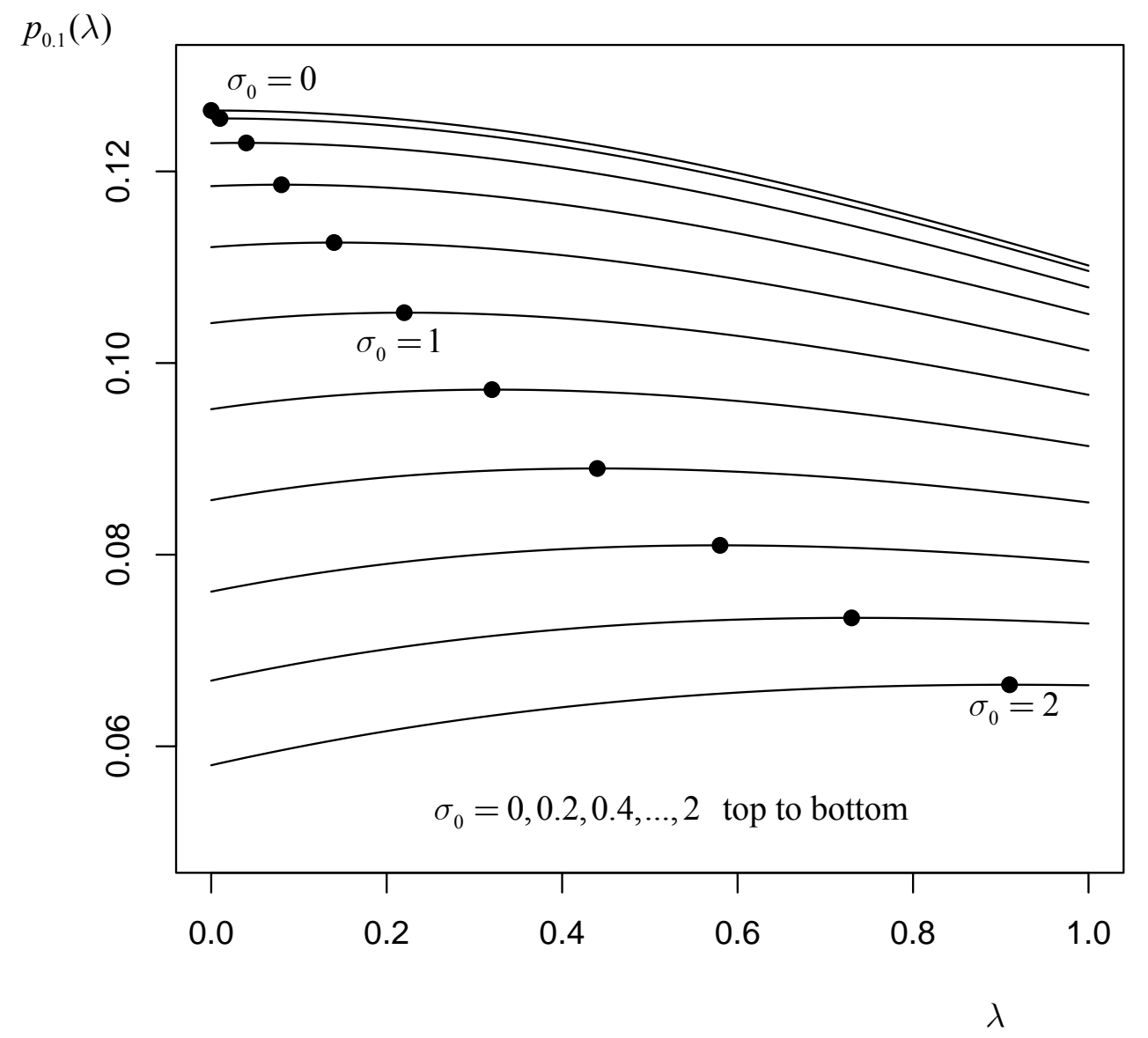

\section{Comparison with Bayesian inference}

It may appear that the tail function methodology is Bayesian (see Lee, 1989) since it requires the specification of a prior distribution. However, this is not so, because nowhere does that methodology involve a posterior calculation. The resulting CI's are all frequentist in the usual sense. It may then be asked why Bayesian posterior intervals should not be used instead, considering that with informative priors they can result in far greater width reductions than those which have been mentioned (e.g. an AGMPDL of $16.1 \%$ when $\alpha=0.05)$. 
The answer is that a Bayesian posterior interval may fail to contain the target parameter with the required probability if that parameter happens to be outside 'the most probable region'. In contrast, frequentist CI's have the correct coverage probabilities for all possible values of the target parameter. Tail functions provide a tool whereby prior information can be usefully incorporated into the inferential process without sacrificing this very important feature of the frequentist approach.

\section{Example 2}

Suppose that $\mu$ 's prior is $N\left(\mu_{0}, \sigma_{0}^{2}\right)$ (see Note 4). Then it is a standard result that $\mu$ 's posterior is $N\left(\mu_{*}, \sigma_{*}^{2}\right)$, where $\mu_{*}=(1-k) \mu_{0}+k \bar{x}, \quad \sigma_{*}^{2}=k \sigma^{2} / n \quad$ and $k=1 /\left\{1+\sigma^{2} /\left(n \sigma_{0}^{2}\right)\right\}$ (the 'credibility factor'). It follows that $\mu$ 's $1-\alpha$ highest posterior density region (HPDR) is $H(\bar{x})=\left(\mu_{*} \pm z_{\alpha / 2} \sigma_{*}\right)$. The length of this interval is $2 z_{\alpha / 2} \sigma_{*}$ and so the associated PDL is $1-2 z_{\alpha / 2} \sigma_{*} /\left\{2 z_{\alpha / 2} \sigma / \sqrt{n}\right\}=1-\sqrt{k}$.

For example, suppose that $\mu_{0}=0$ and $n=\sigma=1$. Then for $\sigma_{0}=0,1,2$, respectively, we find that $k=0,0.5,0.8$; and the associated PDL's are 1, 0.293 and 0.106 . These results may be compared to the corresponding PEPDL's in Figure 8, whose maxima are $0.126,0.105$ and 0.066 . Thus the Bayesian 95\% HPDR is in every case shorter in expectation than the optimal modified $95 \%$ CI defined by tail function (12), $\eta=\mu_{0}$ and $\delta=0.1$.

Let us now examine the frequentist coverage properties of $\mu$ 's Bayesian HPDR. It can be shown that the conditional probability of that HPDR containing $\mu$ is

$$
P_{\mu}=P(\mu \in H(\bar{X}) \mid \mu)=\Phi\left(\frac{\left(\mu-\mu_{0}\right)(1-k)}{k \sigma / \sqrt{n}}+\frac{z_{\alpha / 2}}{\sqrt{k}}\right)-\Phi\left(\frac{\left(\mu-\mu_{0}\right)(1-k)}{k \sigma / \sqrt{n}}-\frac{z_{\alpha / 2}}{\sqrt{k}}\right)
$$

(see Note 6). Figure 9 shows this probability when $\alpha=0.05, \mu_{0}=0$ and $n=\sigma=$ $\sigma_{0}=1$, and also $\mu$ 's prior pdf, $f(\mu)=\phi\left(\left(\mu-\mu_{0}\right) / \sigma_{0}\right) / \sigma_{0}$. We see that $P_{\mu} \longrightarrow 0$ as $\mu \longrightarrow \pm \infty$. Also, $P_{\mu}>0.95$ if and only if $-1.12<\mu<1.12$; and therefore $P\left(P_{\mu}>0.95\right)=0.737$. Thus the frequentist coverage probability of the Bayesian $95 \%$ HPDR falls severely short of the required $95 \%$ level if $\mu$ happens to be far from its prior mean, $\mu_{0}$; and the prior probability of that coverage probability being at least $95 \%$ is only $74 \%$. In contrast, any $95 \%$ CI in the class defined by tail function (12) contains $\mu$ with probability exactly $95 \%$ for all possible values of $\mu$. 
Page 18 of 42

Figure 9 Prior density of $\mu$ and coverage probabilities of $\mu$ 's HPDR

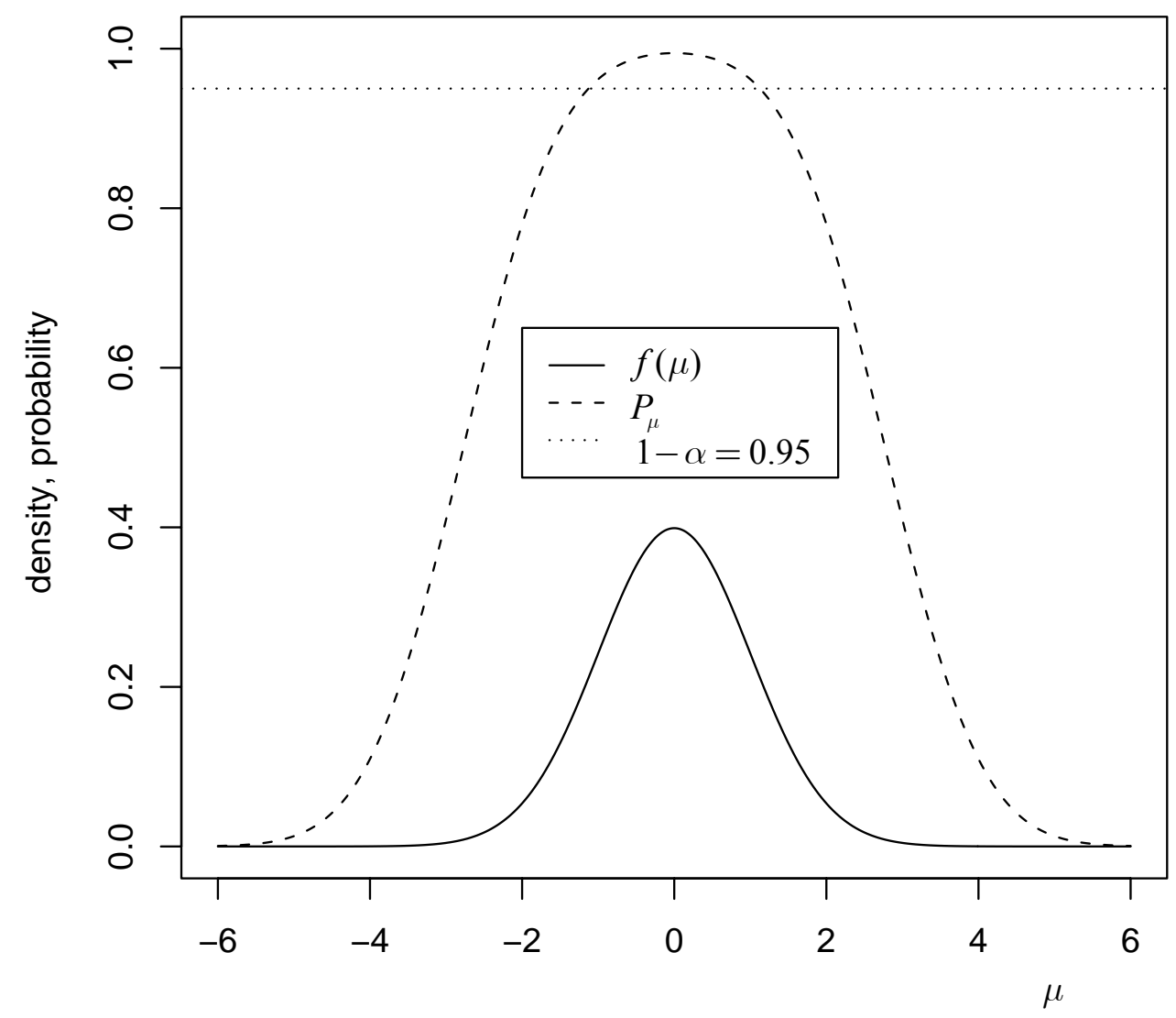


Page 19 of 42

\section{Inference on a normal mean with unknown variance}

Consider the scenario in Section 4 but with the normal variance $\sigma^{2}$ unknown rather than known. In that case the standard classical approach is to make use of the result

$$
Y=\frac{\bar{X}-\mu}{S / \sqrt{n}} \sim t(n-1), \text { where } S^{2}=\frac{1}{n-1} \sum_{i=1}^{n}\left(X_{i}-\bar{X}\right)^{2} \text { is the sample variance, }
$$

and thereby construct a $1-\alpha$ CI for $\mu$ of the form $\left(\bar{x} \pm t_{\alpha / 2}(n-1) s / \sqrt{n}\right)$, where $t_{p}(v)$ denotes the upper $p$-quantile of the $t$-distribution with $v$ degrees of freedom.

To find the upper bound of a modified $1-\alpha$ CI for $\mu$ in this case, we choose a suitable starting value $\mu_{0}$ and repeatedly calculate $\mu_{j+1}=\mu_{j}-\psi\left(\mu_{j}\right) / \psi^{\prime}\left(\mu_{j}\right)$ until convergence, where now:

$$
\begin{aligned}
& \psi(\mu)=F_{t(n-1)}\left(\frac{\bar{x}-\mu}{s / \sqrt{n}}\right)-\alpha \tau(\mu) \\
& \psi^{\prime}(\mu)=f_{t(n-1)}\left(\frac{\bar{x}-\mu}{s / \sqrt{n}}\right)\left(\frac{-1}{s / \sqrt{n}}\right)-\alpha \tau^{\prime}(\mu) .
\end{aligned}
$$

Here, $F_{t(n-1)}($.$) and f_{t(n-1)}($.$) denote the cdf and pdf of the t$ distribution with $n-1$ degrees of freedom ( $c f$. (14) and (15)). The lower bound can be found in the same way but with $\alpha \tau(\mu)$ in (23) replaced by $1-\alpha+\alpha \tau(\mu)$, and with (24) unchanged.

When $\lambda=0$, the confidence bounds are given by (20) with each $z_{p}$ replaced by $t_{p}(n-1)$, and with $\sigma$ in each $\kappa$ replaced by $s$. Similar modifications apply to all the other equations in Section 4, such as that for the MPIL at (18). As in Section 4, it is a good practise to specify $\eta$ as $\mu$ 's prior mean, $\mu_{0}$, and to set $\delta$ equal to the value implied by a desired MPIL.

If an optimal value of $\lambda$ is desired, we first define the PEPDL in this context as

$$
p_{\delta}(\lambda)=E D_{\delta, \lambda}(\bar{X}, S)=\int_{-\infty}^{\infty} \int_{0}^{\infty} \int_{-\infty}^{\infty} \int_{0}^{\infty} D_{\delta, \lambda}(\bar{x}, s) f(\bar{x}, s \mid \mu, \sigma) f(\mu, \sigma) d \bar{x} d s d \mu d \sigma
$$

(cf. (21)), where $D_{\delta, \lambda}(\bar{x}, s)=1-W_{\delta, \lambda}(\bar{x}, s) / W(s)$ is the PDL $(c f .(16)), W_{\delta, \lambda}(\bar{x}, s)$ is the width of the modified CI, $W(s)=2 t_{\alpha / 2}(n-1) s / \sqrt{n}$ is the width of the ordinary CI, $f(\bar{x}, s \mid \mu, \sigma)$ is the joint conditional pdf of $\bar{X}$ and $S$, and $f(\mu, \sigma)$ is the joint prior pdf of $\mu$ and $\sigma$. For more details see Note 7 . 
As in Section 4 our task is to find the value of $\lambda$ which maximizes $p_{\delta}(\lambda)$. Fortunately, (25) can easily be approximated via Monte Carlo for any given value of $\lambda$. One way is to draw values of $\mu$ and $\sigma$ from their joint prior distribution, generate a random sample of size $n$ from the associated $N\left(\mu, \sigma^{2}\right)$ distribution, calculate the corresponding standard and new CI's, repeat many times, and each time record the PDL. The result will be a random sample, $d_{1}, \ldots, d_{J}$, from the prior distribution of the PDL. An unbiased point estimate of and (ordinary) 95\% CI for the PEPDL, $p_{\delta}(\lambda)$, are then $\bar{d}=J^{-1} \sum_{j=1}^{J} d_{j}$ and $\left(\bar{d} \pm 1.96 s_{d} / \sqrt{J}\right)$, where $s_{d}^{2}=(J-1)^{-1} \sum_{j=1}^{J}\left(d_{j}-\bar{d}\right)^{2}$. Repeating this whole process using many different values of $\lambda$ will lead to an estimate of the value $\lambda_{0}$ which maximises $p_{\delta}(\lambda)$.

\section{Example 3}

Suppose that we are about to sample $n=4$ observations from the $N\left(\mu, \sigma^{2}\right)$ distribution where $\sigma^{2}$ is unknown and then construct a $95 \%$ CI for $\mu$. We want the CI to be shorter than the standard $95 \%$ CI, but don't want to risk it being more than $10 \%$ longer. Solving $\left\{t_{\alpha \delta}(3)+t_{\alpha(1-\delta)}(3)\right\} /\left\{2 t_{\alpha / 2}(3)\right\}-1=0.1$ with $\alpha=0.05$ (see (18)), we find that the appropriate value of $\delta$ is 0.2227 .

Suppose that our prior distributions for $\mu$ and $\sigma$ are independently $N\left(\mu_{0}, \sigma_{0}^{2}\right)$ and $\operatorname{Gamma}(a, b)$, where $\mu_{0}=50, \sigma_{0}=5, a=100$ and $b=10$ (so that $E \sigma=a / b=10$ ). These priors are shown in Figure 10. Applying the above Monte Carlo procedure with $\eta=50, \delta=0.2227, J=1000$ and each $\lambda=0,1,2, \ldots, 20$, we obtain the estimates and (ordinary) 95\% CI's for the PEPDL shown in Figure 11. We see that there is little gain to be had from refining the search, and decide to take $\lambda=0$. Figure 11 reveals that if our priors are 'correct' then we can expect the maximal CI defined by $(\eta, \delta, \lambda)$ $=(50,0.2227,0)$ to be about $14.5 \%$ narrower than the standard $95 \%$ CI (see Note 8 ).

We now observe the data and find that $\bar{x}=55$ and $s=17$. Hence by (20) the maximal $95 \% \mathrm{CI}$ for $\mu$ is $\left(55 \pm t_{0.05(1-0.2227)}(3) 17 / \sqrt{4}\right)=(32.6,77.4)$. In contrast, the ordinary $95 \% \mathrm{CI}$ is $\left(55 \pm t_{0.025}(3) 17 / \sqrt{4}\right)=(27.9,82.1)$. The lengths of these CI's are 44.9 and 54.1. Therefore the new methodology has resulted in an improvement of $17.1 \%$.

Note that this is the maximum improvement which was possible, since by (19) the GMPDL is $G_{0.2227}=1-t_{\alpha(1-0.2227)}(3) / t_{\alpha / 2}(3)=0.171$. Also note that exactly the same improvement would have been achieved had the sample mean, $\bar{x}=55$, been any other number in the interval $\left(50 \pm t_{0.05(1-0.2227)}(3) 17 / \sqrt{4}\right)=(27.6,72.4)$. 
Figure 10 Prior densities of the normal mean and standard deviation

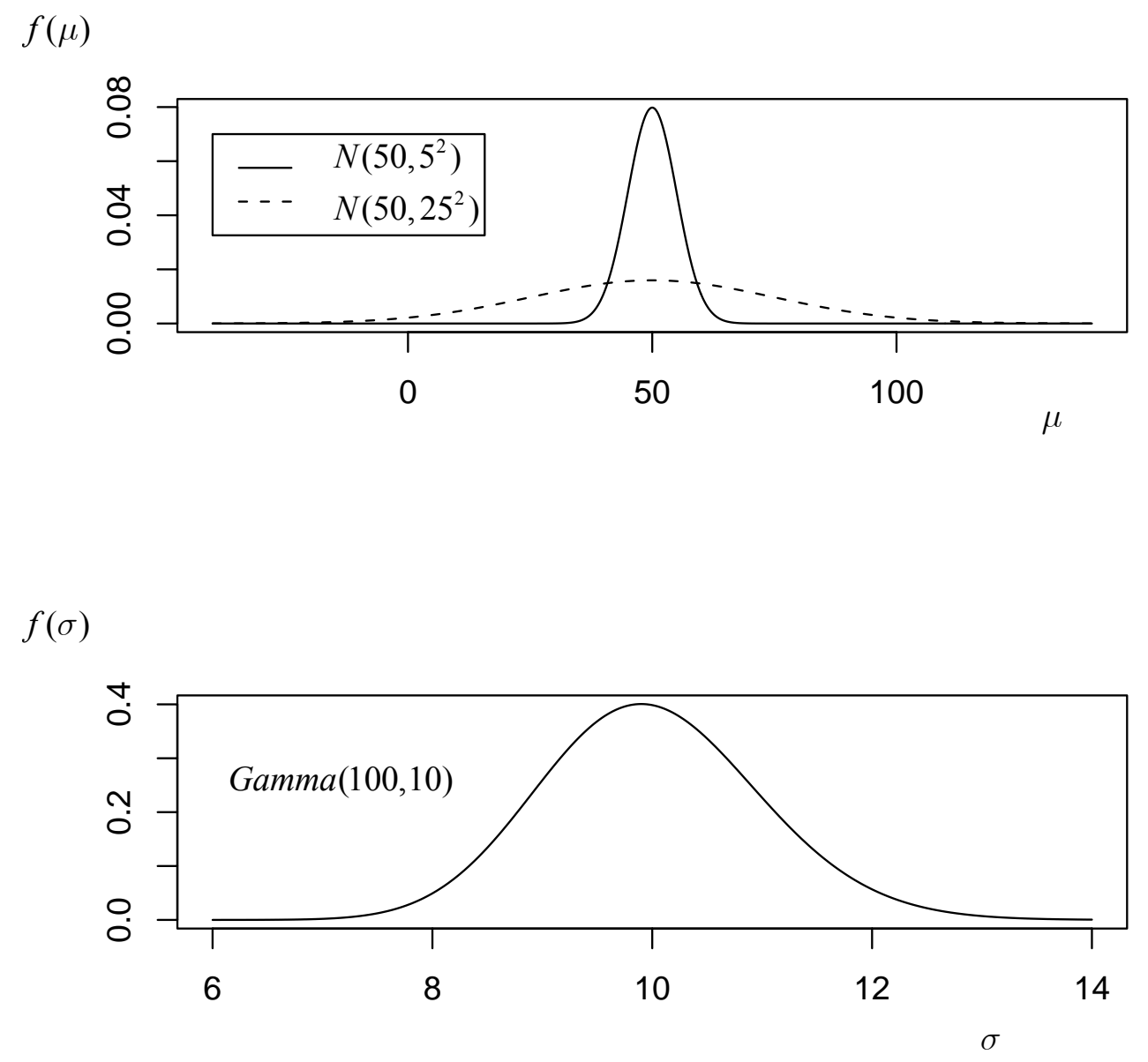


Figure 11 Monte Carlo estimates of the PEPDL when $\sigma_{0}=5$

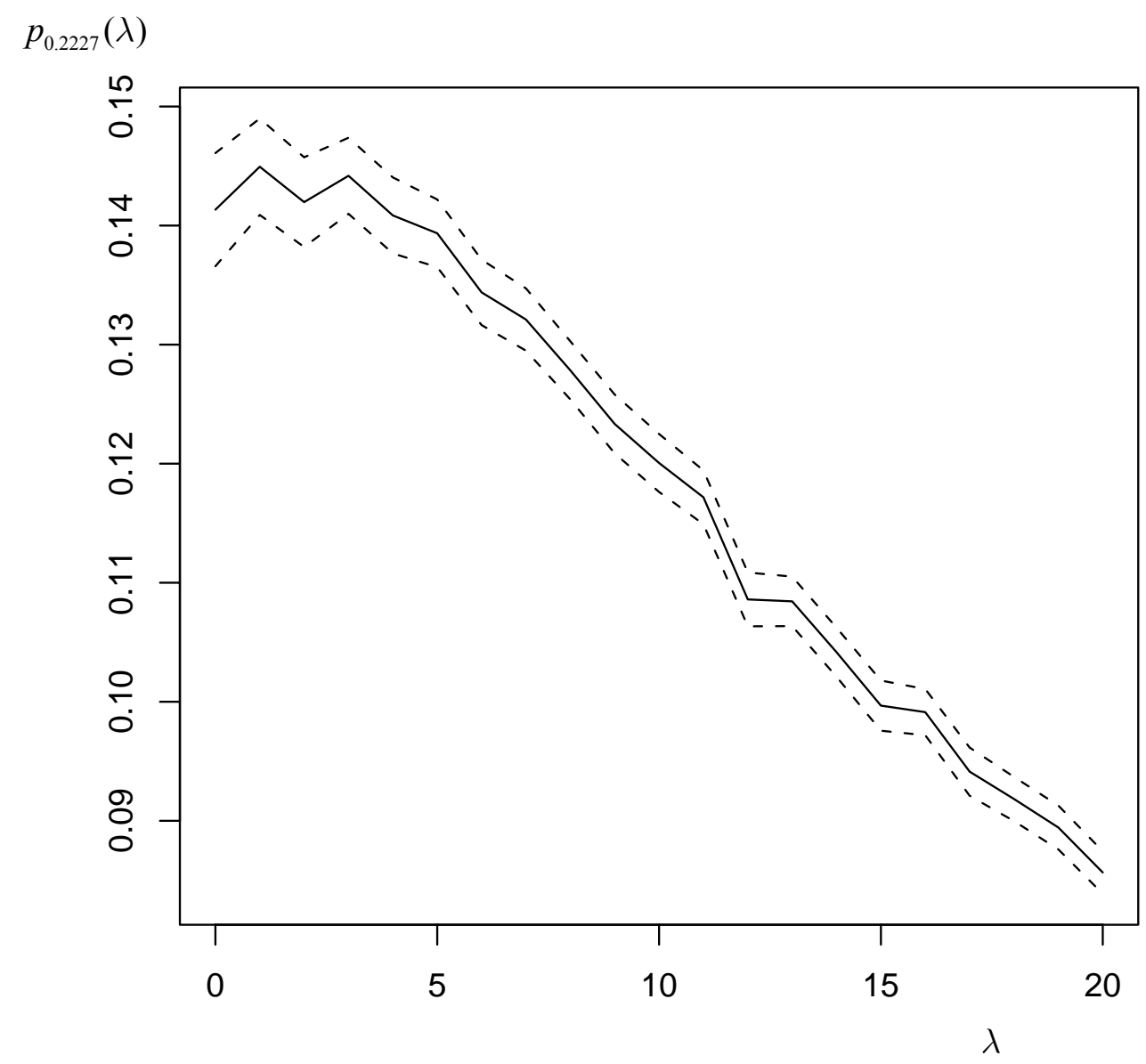

\section{Example 4}

Consider the situation in Example 3, but with $\sigma_{0}=25$ (rather than 5). The corresponding prior on $\mu$ is shown in Figure 10. We repeat the Monte Carlo search procedure, this time over $\lambda=0,5,10, \ldots, 100$, and conclude on the basis of the results shown in Figure 12 that the optimal value of $\lambda$ is about 20 (see Note 9). Applying the Newton Raphson algorithm defined by (23) and (24), with $\eta=50, \delta=0.2227$, $\lambda=20, n=4, \bar{x}=55$ and $s=17$, we find that the new $95 \%$ CI for $\mu$ is $(31.2,78.0)$.

Observe that this interval is slightly wider than the maximal one, $(32.6,77.4)$, and only $13.5 \%$ narrower than the ordinary one, $(27.9,82.1)$. This makes sense because a poorer performance is to be expected with a decrease in prior information. Actually, $13.5 \%$ is well above the $4.5 \%$ improvement which could on the basis of Figure 12 be expected when $\sigma_{0}=25$ and $\lambda=20$. Note that with $\sigma_{0}=25$ and $\lambda=0$, the expected decrease in interval length is only about $2.5 \%$ (see Note 9 ). 
The fact that the actual improvement using $\lambda=0$ is $17.1 \%$ (see Example 3) may be attributed to $\bar{x}$ happening by chance to fall very near $\mu$ 's prior mean, $\mu_{0}=\eta=50$. When $\sigma_{0}$ is 25 (rather than 5), specifying $\lambda$ as 20 (rather than 0 ) may be thought of as taking out insurance - at the cost of a slightly wider interval - against the possibility of a much wider interval resulting due to $\bar{x}$ being far from $\mu_{0}$ (then a more likely outcome).

Figure 12 Monte Carlo estimates of the PEPDL when $\sigma_{0}=\mathbf{2 5}$

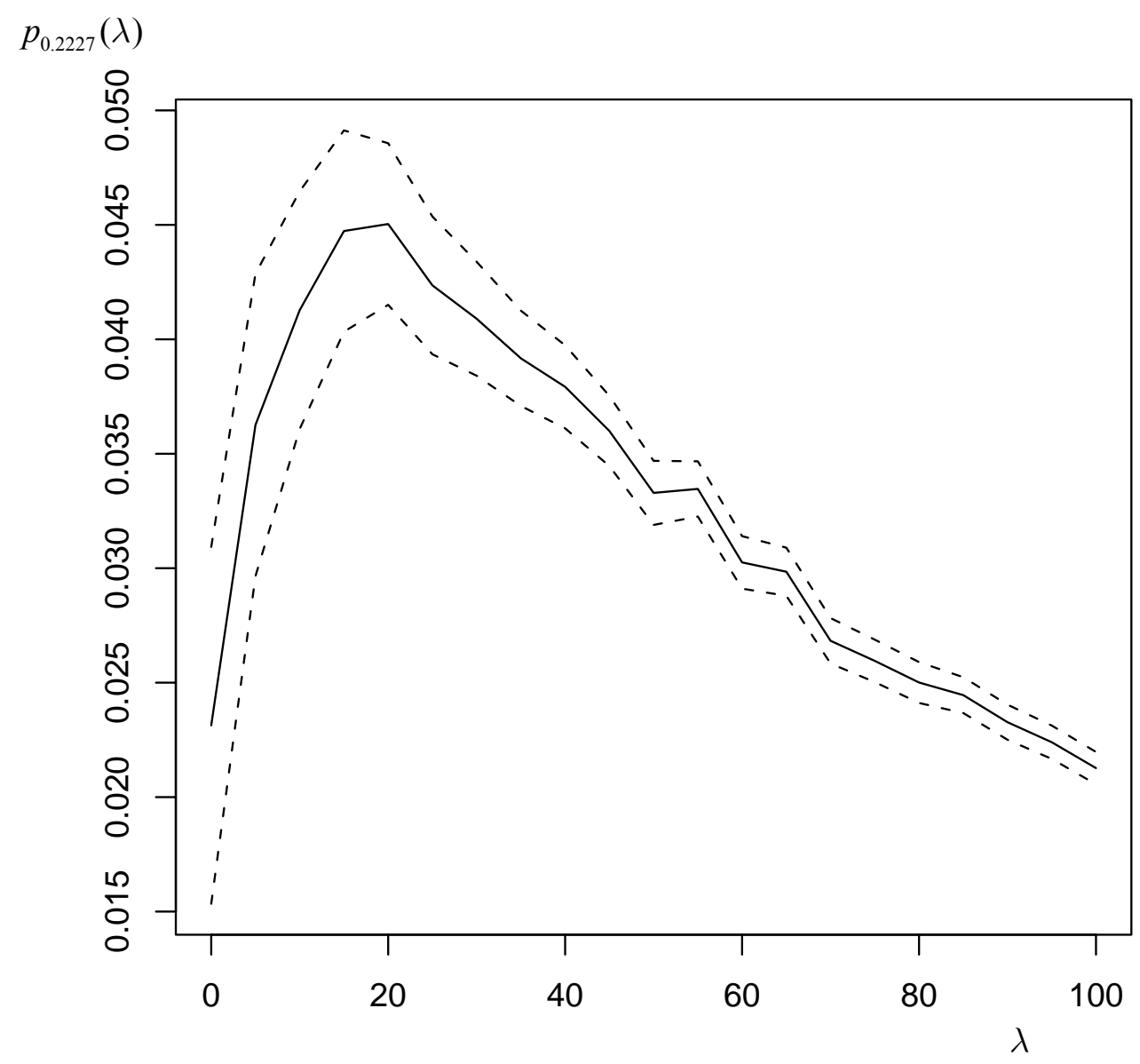




\section{Inference on the binomial proportion}

We will now focus on the problem of estimating a binomial proportion $p$ based on $X$ successes in $n$ Bernoulli trials. It will be shown how tail functions can be used to modify three favourite CI's for $p$, namely the 'standard', Wilson, and ClopperPearson intervals (see Brown et al., 2001), so as to reduce their prior expected lengths. In some cases the modified interval also exhibits better coverage properties.

\section{The 'standard' interval}

We first consider the most commonly used $1-\alpha$ CI for $p$, namely the 'standard' interval. This may be written

$$
\left(\bar{x} \pm z_{\alpha / 2} \sigma / \sqrt{n}\right)
$$

where $\bar{x}=x / n, x$ is the observed value of $X, \sigma^{2}=\bar{x}(1-\bar{x})$, and where bounds less than 0 or greater than 1 are taken as 0 or 1 , respectively. This approximate CI is a consequence of the central limit theorem which, with $\bar{X}=X / n$, implies that

$$
\frac{\bar{X}-p}{\sqrt{\bar{X}(1-\bar{X}) / n}} \stackrel{d}{\longrightarrow} N(0,1) \quad \text { as } n \longrightarrow \infty \text {. }
$$

The lower and upper bounds of (26) are obtained as the values of $p$ for which $\Phi((\bar{x}-p) \sqrt{n} / \sigma)$ equals $1-\alpha / 2$ and $\alpha / 2$, respectively.

We see that this scenario is identical to the one in Section 4 , with $\mu=p$. Thus if prior information regarding $p$ is available, we may replace $1-\alpha / 2$ and $\alpha / 2$ in the last paragraph with $1-\alpha+\alpha \tau(p)$ and $\alpha \tau(p)$, where $\tau(p)$ is given by (12) and suitable choices of $\eta, \delta$ and $\lambda$. Note that $\sigma^{2}=0$ if $x=0$ or $n$, in which case we define the modified standard $\mathrm{CI}$ as the single point 0 or 1 , respectively (i.e. we make no modification to the ordinary standard CI).

\section{Example 5}

Suppose that we are about to conduct a binomial experiment with $n=100$ trials and feel confident that $p$ lies near 0.5 . We consider using a Gaussian standard $95 \% \mathrm{CI}$ with parameters $\eta=0.5$ and $\delta=0.01$. Figure 13 shows the bounds of this CI for all 
possible outcomes, $x=0,1 \ldots, 100$, both when $\lambda=0$ and when $\lambda=0.1$. Also shown are the bounds of the ordinary standard $95 \% \mathrm{CI}(\delta=0.5)$.

Figure 13 Bounds of three standard 95\% CI's when $n=100$ and $\eta=0.5$

$x$

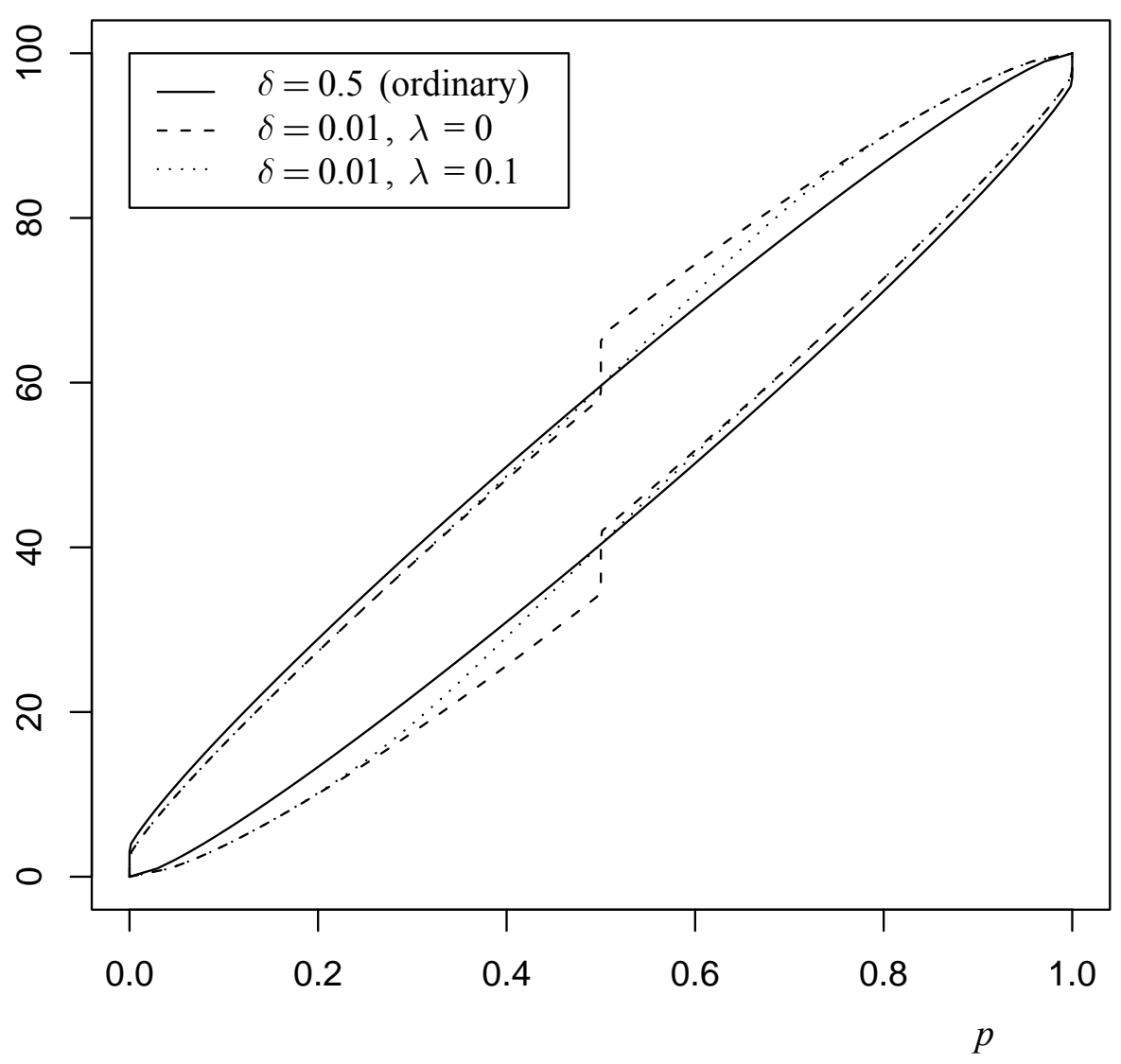

Observe in Figure 13 that the two modified CI's are shorter than the ordinary CI when $x$ is close to 50, corresponding to $p$ being close to $\eta=0.5$. This effect is 'paid for' by the modified CI's being longer for other values of $x$.

Let us now compare the coverage probabilities of the three CI's. These are illustrated in Figure 14, and it is interesting that both of the modified 95\% CI's have coverage properties which are distinctly better than those of the standard $95 \% \mathrm{CI}$. 
Figure 14 Coverage probabilities of three standard 95\% CI's when $n=100$ and $\eta=0.5$
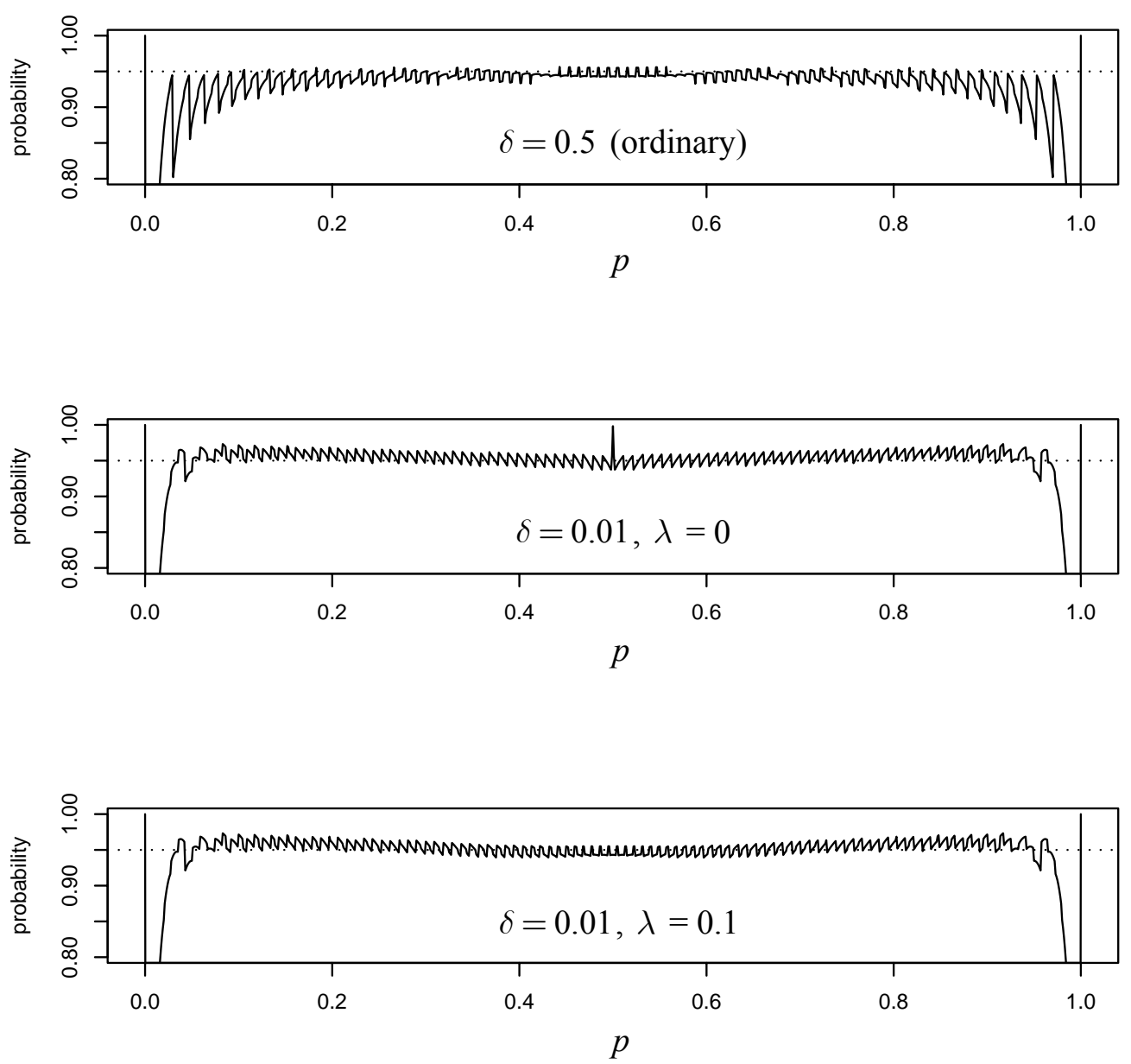

Let us now compare expected lengths. In Figure 15 we see that the maximal CI (defined by $\lambda=0$ ) has lengths which are smaller on average than those of the ordinary CI for values of $p$ between about 0.4 and 0.6 , and larger for values outside that range. The CI defined by $\lambda=0.1$ provides a greater range for which there is improvement over the ordinary interval (roughly 0.3-0.7), and also a smaller maximum expected length ( 0.18 rather than 0.22$)$. However, these improvements come at the expense of it being slightly longer than the maximal CI for $p$ very near 0.5 . 
Figure 15 Expected lengths of three standard 95\% CI's when $n=100$ and $\eta=0.5$

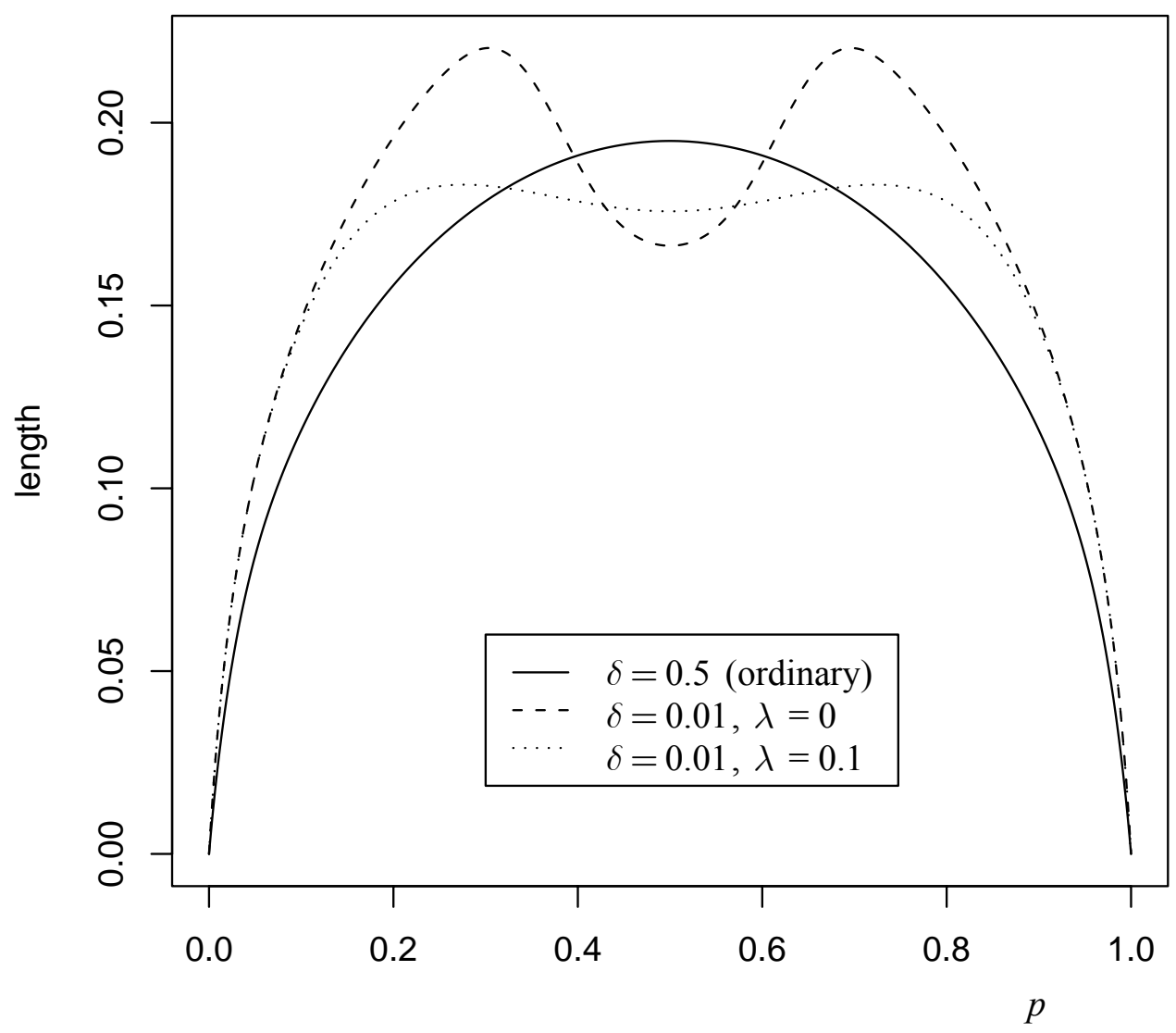

Our final decision regarding which interval to use will depend on the strength of our prior beliefs. For example, if we believe very strongly that $p$ is between 0.45 and 0.55 then a good choice is the maximal CI defined by $(\eta, \delta, \lambda)=(0.5,0.01,0)$. If we believe that $p$ might be anywhere between 0.3 and 0.7 then a 'safer' choice is $(\eta, \delta, \lambda)=(0.5,0.01,0.1)$. If we believe that $p$ could be anywhere from 0.2 to 0.8 , then perhaps it is best to stick with the ordinary standard interval $(\delta=0.5)$, or else investigate other values of $\delta$ and $\lambda$. 


\section{The Wilson interval}

An interval with better coverage properties than the standard interval, especially when $n$ is small, is the Wilson interval (Wilson, 1927), whose bounds are given by:

$$
\left(l_{a}, u_{a}\right)=\left(\frac{\bar{x}+\frac{z_{a}^{2}}{2 n}-z_{a} \sqrt{\frac{\bar{x}(1-\bar{x})}{n}+\frac{z_{a}^{2}}{4 n^{2}}}}{1+\frac{z_{a}^{2}}{n}}, \frac{\bar{x}+\frac{z_{a}^{2}}{2 n}+z_{a} \sqrt{\frac{\bar{x}(1-\bar{x})}{n}+\frac{z_{a}^{2}}{4 n^{2}}}}{1+\frac{z_{a}^{2}}{n}}\right),
$$

where $a=\alpha / 2$. This interval is based on the result

$$
\frac{\bar{X}-p}{\sqrt{p(1-p) / n}} \stackrel{d}{\longrightarrow} N(0,1) \quad \text { as } n \longrightarrow \infty
$$

where the rate of convergence is faster than in (27). It can be shown that if we apply the tail function (12) with $\lambda=0$, then the resulting modified $1-\alpha$ Wilson CI is:

$$
\begin{array}{lll}
\left(l_{\alpha \delta},\right. & \left.u_{\alpha(1-\delta)}\right), & \bar{x}>\eta+d \\
(\eta, & \left.u_{\alpha(1-\delta)}\right), & \eta+c<\bar{x} \leq \eta+d \\
\left(l_{\alpha(1-\delta)},\right. & \left.u_{\alpha(1-\delta)}\right), & \eta-c \leq \bar{x} \leq \eta+c \\
\left(l_{\alpha(1-\delta)},\right. & \eta), & \eta-d \leq \bar{x}<\eta+c \\
\left(l_{\alpha(1-\delta)},\right. & \left.u_{\alpha \delta}\right), & \bar{x}<\eta-d,
\end{array}
$$

where $c=z_{\alpha(1-\delta)} \sqrt{\eta(1-\eta) / n}$ and $d=z_{\alpha \delta} \sqrt{\eta(1-\eta) / n}$. Note that if $\delta=1 / 2$ then the maximal Wilson CI (30) reduces to the ordinary Wilson CI (28), which in turn reduces to the ordinary standard CI (26) in the limit as $n$ tends to infinity.

If $\lambda>0$ then the modified Wilson CI can be obtained via the Newton-Raphson algorithm as follows. The upper bound may be found by choosing a suitable starting value $p_{0}$ and iterating $p_{j+1}=p_{j}-\psi\left(p_{j}\right) / \psi^{\prime}\left(p_{j}\right)$ until convergence, where:

$$
\begin{aligned}
& \psi(p)=\Phi(g(x, p))-\alpha \tau(p) \\
& \psi^{\prime}(p)=\phi(g(x, p)) \frac{\partial}{\partial p} g(x, p)-\alpha \tau^{\prime}(p) \\
& g(x, p)=\frac{\bar{x}-p}{\sqrt{p(1-p) / n}} \text { and } \frac{\partial}{\partial p} g(x, p)=\frac{\bar{x}(p-1 / 2)-p / 2}{\sqrt{p^{3}(1-p)^{3} / n}} .
\end{aligned}
$$


The lower bound can be found in the same way but with $\alpha \tau(p)$ in (31) replaced by $1-\alpha+\alpha \tau(p)$, and with (32) unchanged. Note that this Newton-Raphson algorithm can also be used to find the modified standard CI after replacing (33) with $g(x, p)=(\bar{x}-p) / \sqrt{\bar{x}(1-\bar{x}) / n}$ and $\partial g(x, p) / \partial p=-1 / \sqrt{\bar{x}(1-\bar{x}) / n}$.

\section{Example 6}

Suppose that we are about to conduct a binomial experiment with $n=25$ trials and feel confident that $p$ lies near 0.3 . Since $n$ is small we consider using a modified Wilson $95 \%$ CI with parameters $\eta=0.3$ and $\delta=0.03$, where $\lambda$ is either 0 or 0.15 .

Figures 16-18 provide the same kinds of information as Figures 13-15. In Figure 18 we see that when $p$ is near 0.3 , the maximal Wilson CI (defined by $\lambda=0$ ) provides greater gains in expected length over the standard Wilson CI than the one defined by $\lambda=0.15$, and that this comes at the expense of it being more 'risky' overall. Note that the coverage probabilities of the two modified intervals in Figure 17 are asymmetric, unlike in Figure 14. This is because $\eta$ equals 0.3 and not 0.5 . 
Figure 16 Bounds of three Wilson 95\% CI's when $n=25$ and $\eta=0.3$

$x$

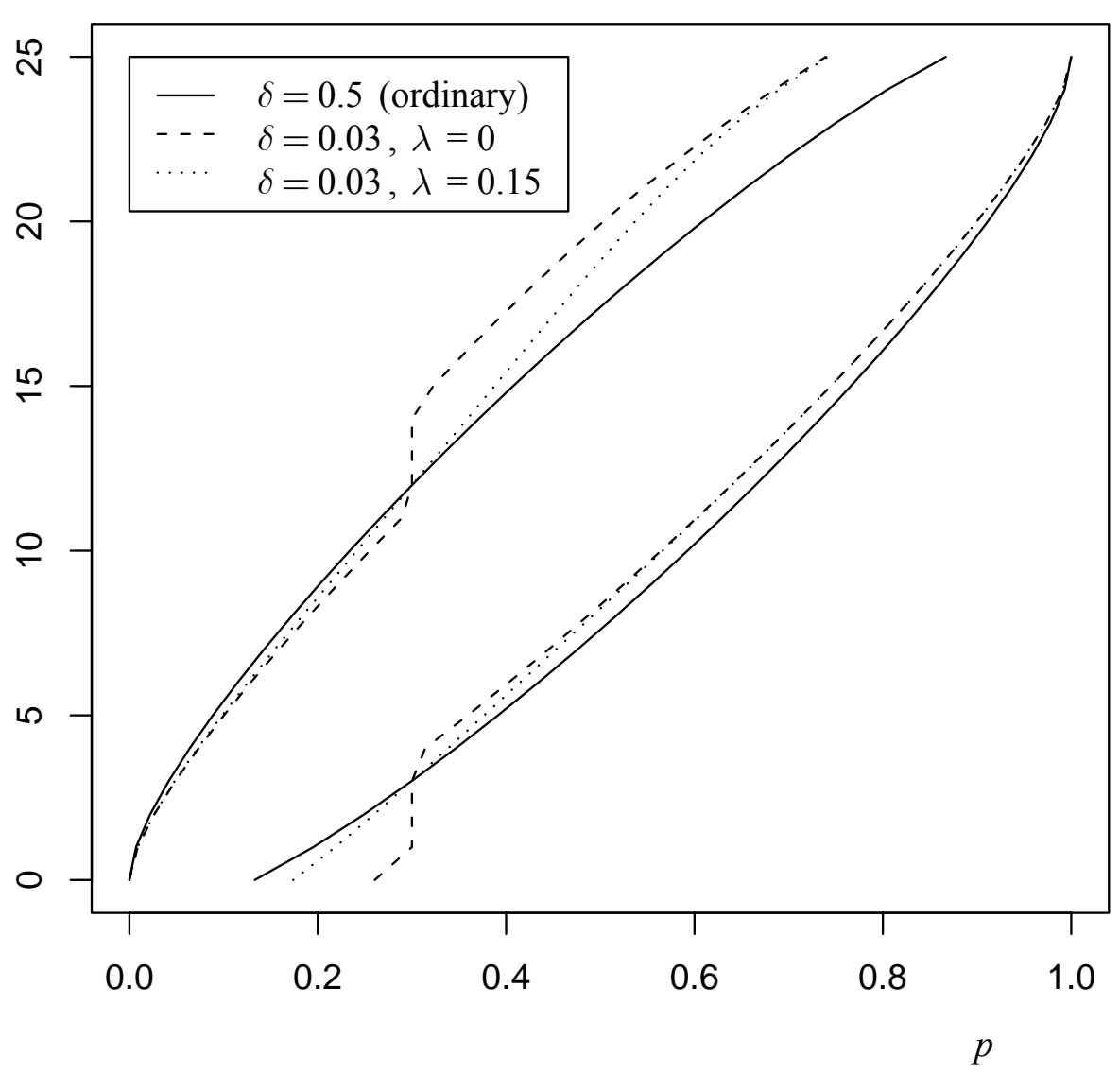


Figure 17 Coverage probabilities of three Wilson 95\% CI's when $n=25$ and $\eta=\mathbf{0 . 3}$
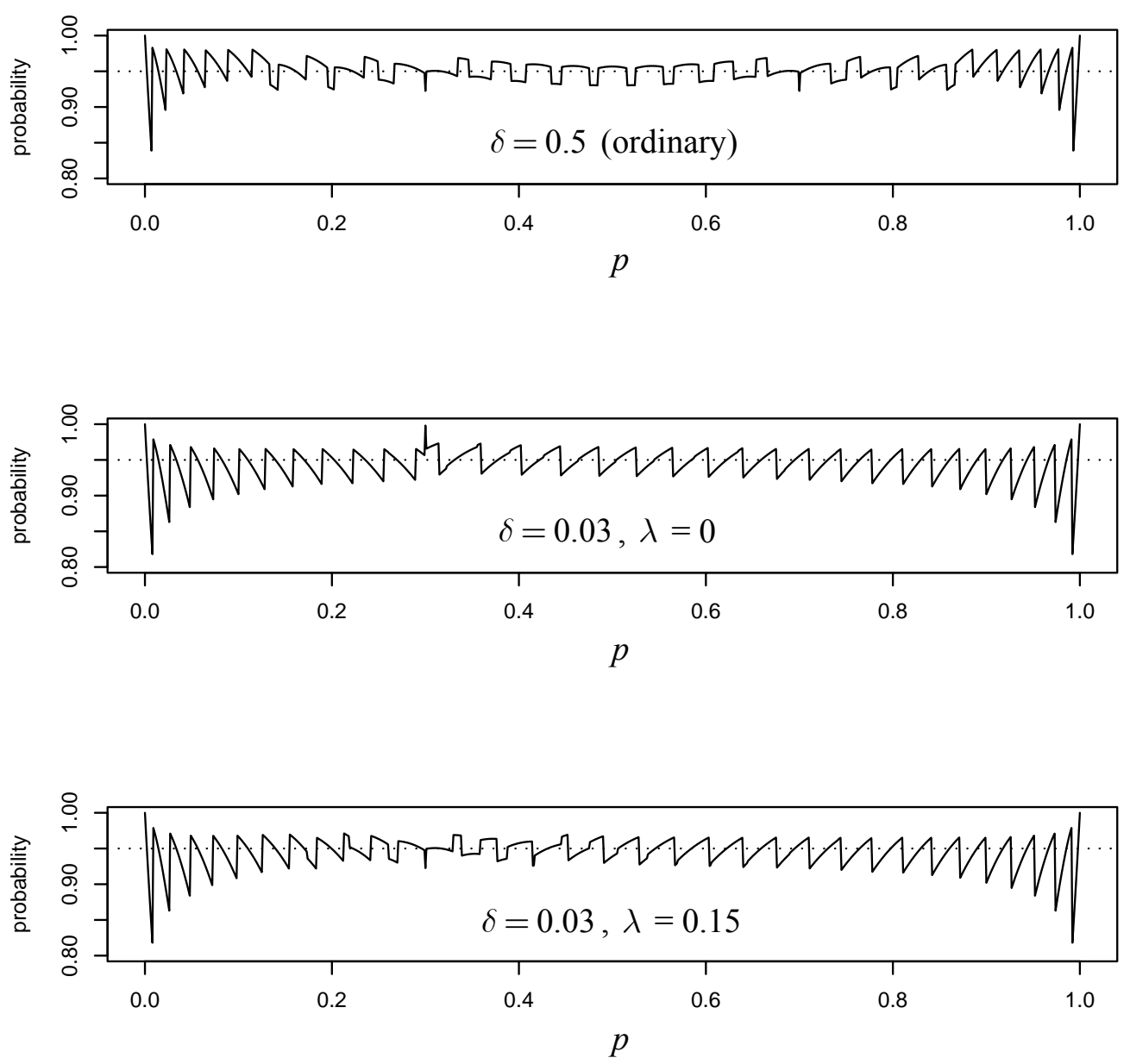
Figure 18 Expected lengths of three Wilson 95\% CI's when $n=25$ and $\eta=\mathbf{0 . 3}$

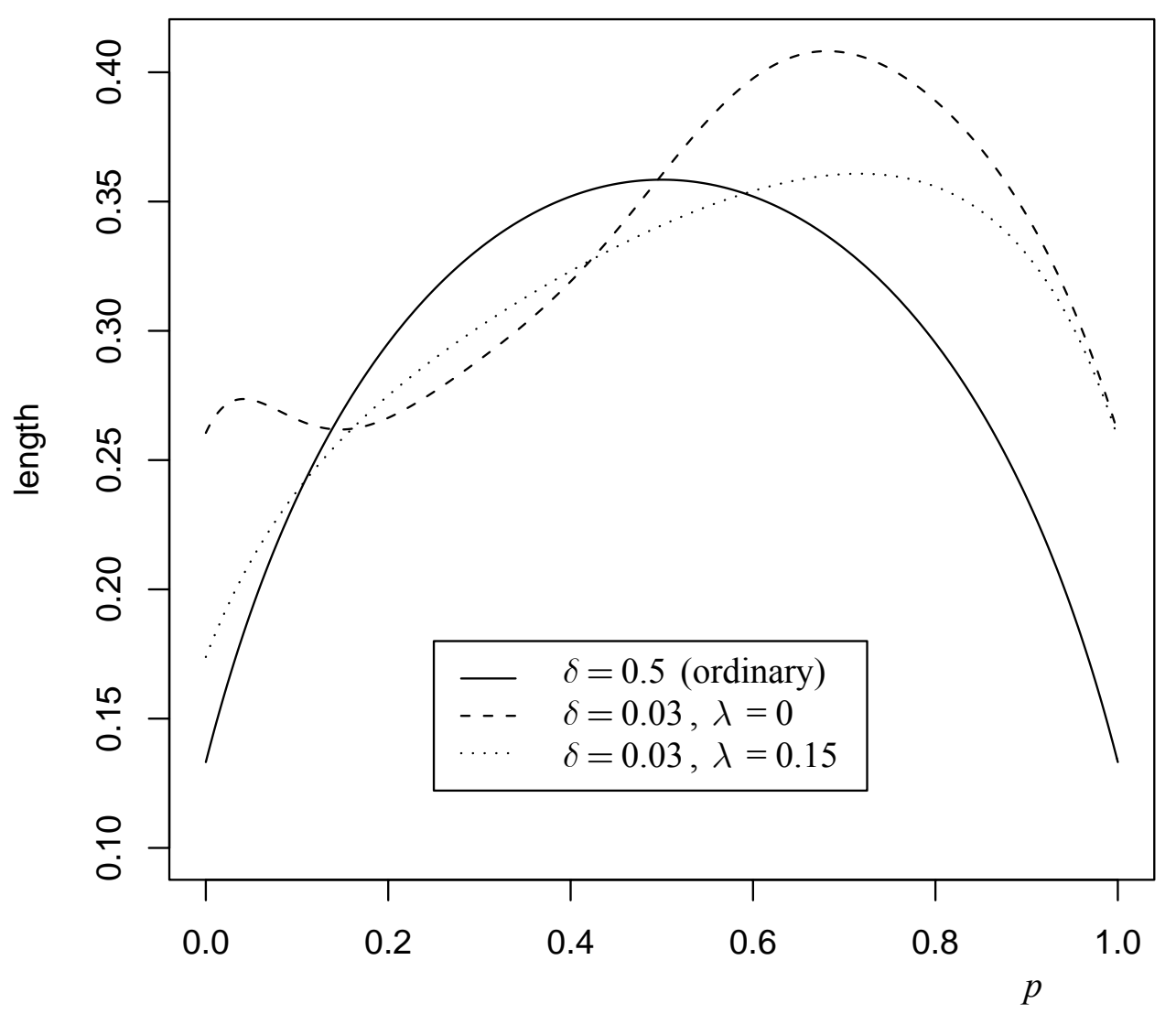




\section{The Clopper-Pearson interval}

As is evident from Figures 14 and 17, the coverage probabilities of the standard and Wilson intervals are only approximately equal to the desired level, $1-\alpha$. One CI which contains $p$ with probability at least $1-\alpha$ is the so-called 'exact' ClopperPearson (CP) CI, after Clopper and Pearson (1934). This interval may be written $(L(a), U(a))$ where, with $a=\alpha / 2, L(a)$ and $U(a)$ are the solutions in $p$ of the equations:

$$
\begin{aligned}
& P(X \geq x ; p)=a \\
& P(X \leq x ; p)=a,
\end{aligned}
$$

respectively, except that $L(a)=0$ if $x=0$ and $U(a)=1$ if $x=n$. It can be shown that $L(a)=\operatorname{Beta}(a ; x, n-x+1)$ and $U(a)=\operatorname{Beta}(1-a ; x+1, n-x)$, where $\operatorname{Beta}(q ; r, s)$ denotes the (lower) $q$ quantile of the beta distribution with parameters $r$ and $s$.

A problem with the $\mathrm{CP}$ CI is that it tends to be wider than necessary, and recently Blaker (2000) has modified it so as to produce a new 'gold standard' that is uniformly shorter on average whilst still being 'exact'. Moreover, Blaker's interval has an attractive nesting condition which is absent from some earlier 'exact' CI's, for example those developed by Blyth and Still (1983) and Casella (1986).

The tail function methodology can also be applied in this context. Given a tail function $\tau(p)$, the modified Clopper-Pearson $\mathrm{CI}$ is defined as $(l, u)$, where $l$ and $u$ are the solutions in $p$ of the equations:

$$
\begin{aligned}
& P(X \geq x ; p)=\alpha(1-\tau(p)) \\
& P(X \leq x ; p)=\alpha \tau(p)
\end{aligned}
$$

respectively, except that once again $l=0$ if $x=0$ and $u=1$ if $x=n$. These equations can be solved via the Newton-Raphson algorithm in the usual manner, after noting that $P(X \leq x ; p)=\sum_{t=0}^{x} f_{X}(t ; p)$ where $f_{X}(t ; p)=C_{t}^{n} p^{t}(1-p)^{n-t}$. The calculations can also be facilitated by noting certain relationships between the binomial and $\mathrm{F}$ distributions, and by interpreting CP CI's, both ordinary and modified, as a natural byproduct of randomised confidence interval theory. For more details see Puza and O’Neill (2004). 
An important special case is where the tail function is a step function, in which case the bounds of the modified CP CI can be written in terms of the ordinary CP CI. For example, suppose that the tail function is Gaussian (12) with $\lambda=0$. Then the bounds of the associated 'maximal' CI are:

$$
\begin{aligned}
& l=\left\{\begin{array}{cc}
L(\alpha(1-\delta)) & \text { if } L(\alpha(1-\delta)) \leq \eta \\
L(\alpha \delta) & \text { if } L(\alpha \delta) \geq \eta \\
\eta & \text { otherwise }
\end{array}\right. \\
& u=\left\{\begin{array}{cc}
U(\alpha(1-\delta)) & \text { if } U(\alpha(1-\delta)) \geq \eta \\
U(\alpha \delta) & \text { if } U(\alpha \delta) \leq \eta \\
\eta & \text { otherwise }
\end{array}\right.
\end{aligned}
$$

These equations can easily be generalized to accommodate any number of steps in the tail function. For an example, see Note 10.

\section{Example 7}

Suppose that we are about to conduct a binomial experiment with only $n=10$ trials, where the goal is an 'exact' $95 \%$ CI for $p$, whose value is believed to be small and certainly less than 0.4. We consider five candidates: the standard CP CI, the Blaker $\mathrm{CI}$, the one-sided CP CI $\left(0, U_{\alpha}\right)$, and the modified CP CI's defined by $(\eta, \delta, \lambda)$ $=(0.2,0,0)$ and $(0.25,0,0)$. Note that the one-sided CI can also be thought of as a modified CP CI defined by $(\eta, \delta, \lambda)=(0,0,0)$, or equivalently, by $\tau(p)=1$.

Figures 19-21 show the same diagnostics as in previous examples, but for the above five CI's. We see in Figure 21 that in terms of expected length, the modified CI defined by $\eta=0.25$ is clearly the best of the five intervals for all $p$ less than 0.4 . In particular, it is everywhere narrower on average than the one-sided CI, except at $p=0$, where the two intervals have exactly the same expected length. For further discussion see Note 11. 
Figure 19 Bounds of five 'exact' 95\% CI's when $\boldsymbol{n}=\mathbf{1 0}$ and $\delta=\lambda=\mathbf{0}$

$x$

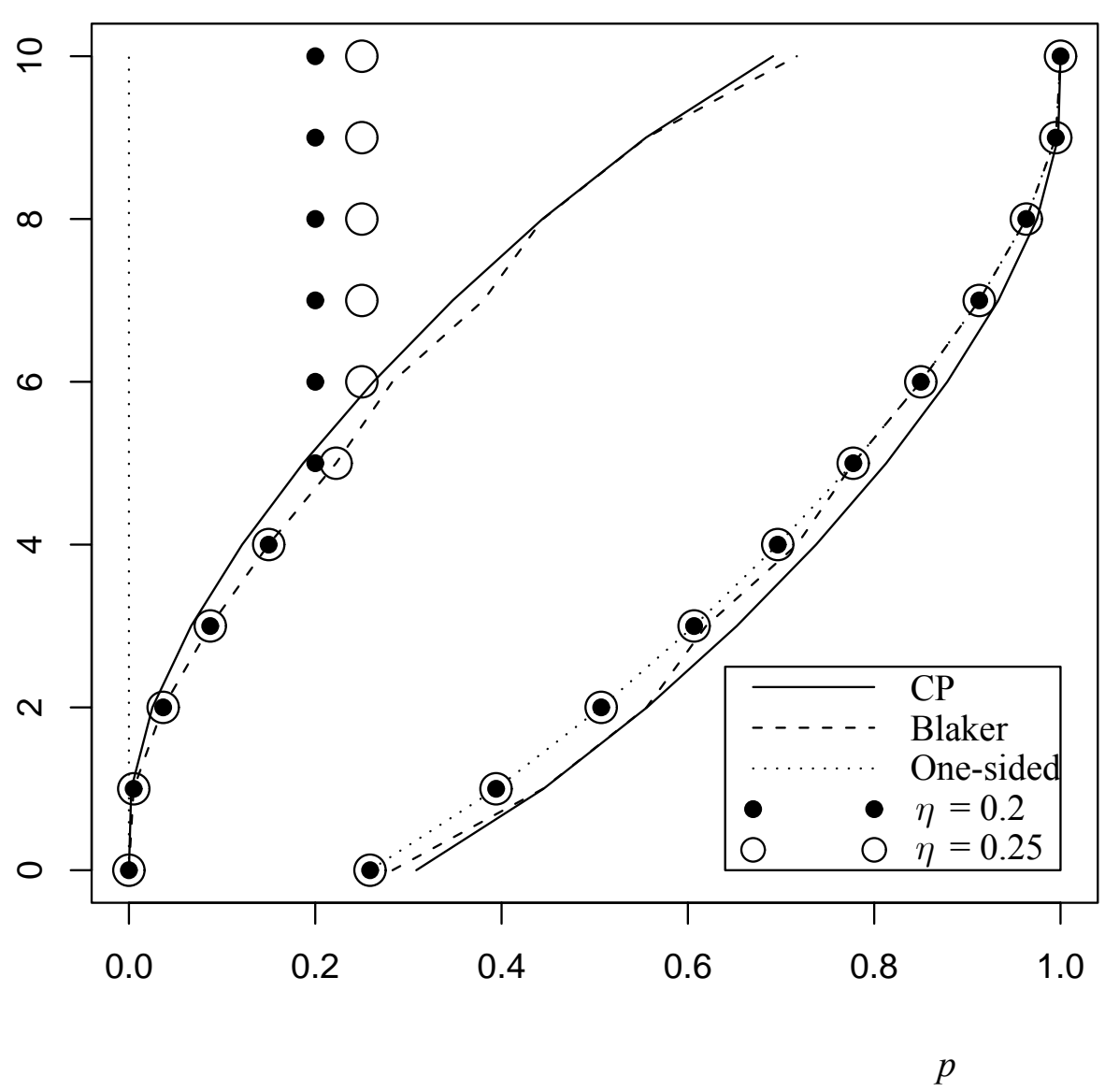


Figure 20 Coverage probabilities of five 'exact' 95\% CI's when $n=10$ and $\delta=\lambda=\mathbf{0}$

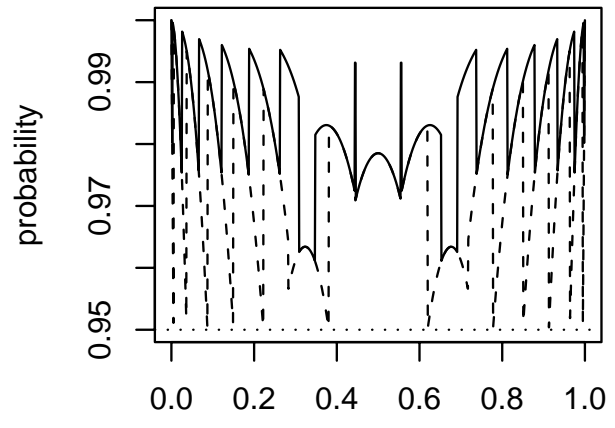

Ordinary CP (solid lines) and Blaker (dashed lines)

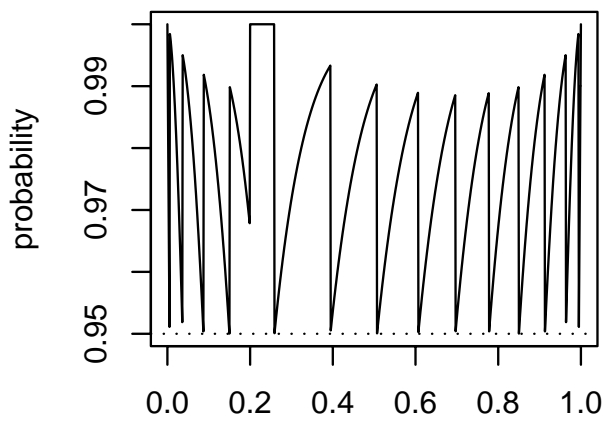

Modified CP $\eta=0.2$

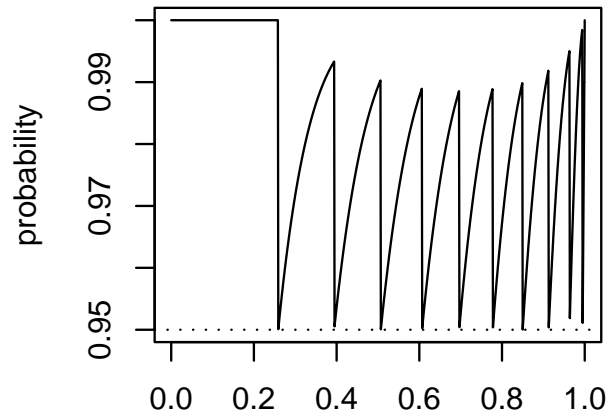

One-sided CP $(\eta=0)$

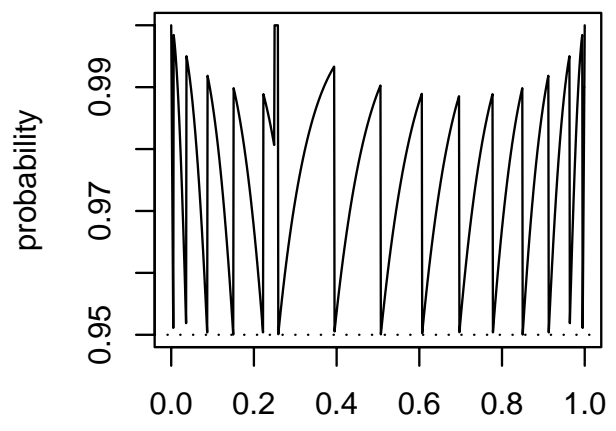

Modified CP $\eta=0.25$ 
Figure 21 Expected lengths of five 'exact' 95\% CI's when $\boldsymbol{n}=10$ and $\delta=\lambda=\mathbf{0}$

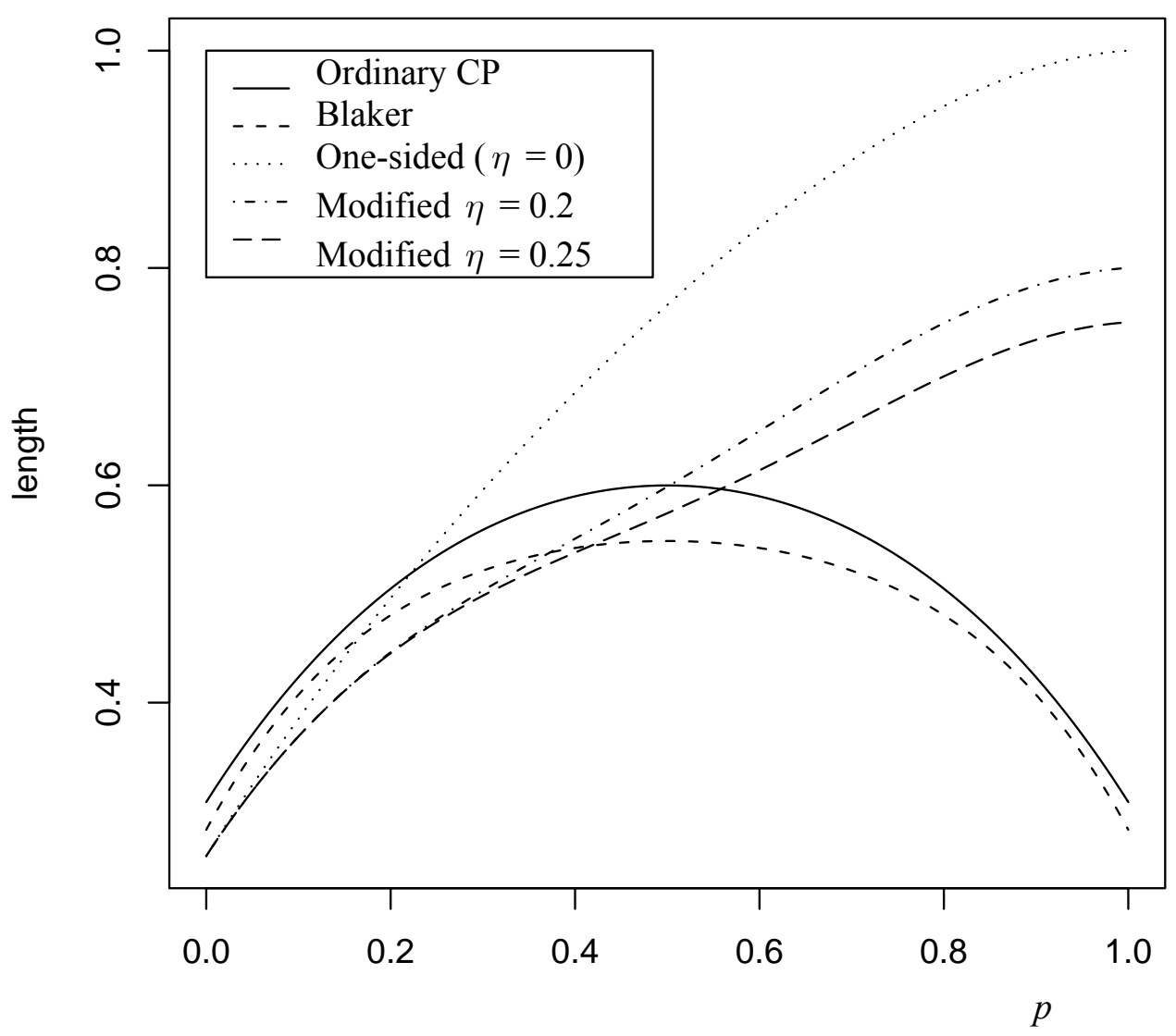


Page 38 of 42

\section{Summary and discussion}

In this paper we have shown how confidence intervals can be constructed using tail functions. Classical methods may be thought of as a special case of the new theory, with all tail functions taken as constant. Focussing on the normal mean and binomial proportion, we have provided several examples of how a suitable choice of tail function can lead to a CI which is shorter in expectation than its 'ordinary' or classical counterpart. Such improvements rely on prior information being available and are most effective when that information is strong.

The tail function methodology provides an attractive frequentist alternative to the Bayesian approach when it is desired that the coverage probability be correct for all values of the target parameter. Also, it has the advantage of not requiring prior information to be expressed in an exact way. For example, if we are about to conduct a binomial experiment with 10 trials and know only that the binomial proportion is less than 0.4 then a good tail function can be found without any additional information (see Example 7). In contrast, the Bayesian approach requires a specific prior distribution, such as the beta, in order to proceed.

A useful observation is that a modified CI tends to be relatively short on average for values of the target parameter at which the tail function is steep. This fact provides a guide to choosing the optimal tail function in any situation. One important special case is where the tail function consists of a single step. The implied CI can often be calculated easily, without the Newton-Raphson algorithm (see (20), (30) and (35)). Moreover, this case allows for the maximum possible reduction in width and is a good choice in many situations. This suggests that we give special consideration to tail functions which consist only of steps (see Note 10)). We leave these and other tail functions as a topic for future research.

It should be kept in mind that there is a price to be paid for a CI with smaller expected width, namely the risk that it will be longer if the data happens to be extreme or the prior has been misspecified. Thus the proposed methodology involves an element of gambling. Also, the choice of tail function should not be allowed to depend on the observed data. If it does, then any reduction in interval width may be illusory due to a deflated coverage effect (see Note 12). Ideally, the exact form of the CI should be specified before the data have been observed. Note that this advice applies equally in any situation where there are two or more ways to construct an interval estimate. 
Page 39 of 42

\section{Notes}

1. Each of the two pairs of lines in Figure 2 also satisfy the following equations:

$$
\begin{array}{ll}
\bar{x}=\mu+\Phi^{-1}(1-\alpha+\alpha \tau(\mu)) \sigma / \sqrt{n} & \text { (upper line) } \\
\bar{x}=\mu+\Phi^{-1}(\alpha \tau(\mu)) \sigma / \sqrt{n} & \text { (lower line). }
\end{array}
$$

The continuous lines correspond to $\tau(\mu)=1 / 2$, and the dashed ones to (12) with $\eta=50, \delta=1 / 8$ and $\lambda=10$. This simple way to graph interval bounds follows from (7), (8) and (9) after putting $\theta=\mu, g(x, \theta)=y=(\bar{x}-\mu) \sqrt{n} / \sigma$ and $F_{Y}(y)=\Phi(y)$.

2. The equation $I_{\delta}=I$ can be solved via Newton-Raphson as follows. Let $h(\delta)=I_{\delta}-I$ and $h^{\prime}(\delta)=\alpha\left(z_{\alpha \delta}^{\prime}-z_{\alpha(1-\delta)}^{\prime}\right) /\left(2 z_{\alpha / 2}\right)$, where $z_{t}^{\prime}=d z_{t} / d t=-1 / \phi\left(z_{t}\right)$. Then choose a starting value $\delta_{0}$ and repeatedly calculate $\delta_{j+1}=\delta_{j}-h\left(\delta_{j}\right) / h^{\prime}\left(\delta_{j}\right)$ until convergence.

3. Except for values of $\lambda$ near the origin, the MPDL's in Figure 6 are proportionate to $\sigma / \sqrt{n}$. For example, if $(n, \sigma)=(1,1)$ is changed to either $(4,1)$ or $(1,0.5)$ then $M_{0.1,2}=0.092$ changes to 0.056 . At the same time, each GMPDL (e.g. $G_{0.0871}=0.138$ ) stays the same, and the 'plateaus' near the origin become shorter. In general, $M_{\delta, \lambda} \longrightarrow G_{\delta} I(\lambda=0)$ as $\sigma \longrightarrow 0$ or $n \longrightarrow \infty$ (for all $\delta$ ), and $M_{\delta, \lambda} \longrightarrow G_{\delta}$ as $\sigma \longrightarrow \infty$ (for all $\delta$ and $\lambda$ ).

4. One way in which the prior $\mu \sim N\left(\mu_{0}, \sigma_{0}^{2}\right)$ might arise is if a random sample of $n_{0}$ values from the $N\left(\mu, \sigma^{2}\right)$ distributions were previously observed and their average was $\bar{x}_{0}$. Using a flat 'pre-prior' for $\mu$ and standard Bayesian calculations (see Lee, 1989), we may then specify $\mu_{0}=\bar{x}_{0}$ and $\sigma_{0}^{2}=\sigma^{2} / n_{0}$.

5. An equivalent definition for the PEPDL at (21) is $p_{\delta}(\lambda)=\int_{-\infty}^{\infty} D_{\delta, \lambda}(\bar{x}) f(\bar{x}) d \bar{x}$, where $f(\bar{x})=\phi\left(\left(\bar{x}-\mu_{0}\right) / \sigma_{\#}\right) / \sigma_{\#}$ and $\sigma_{\#}^{2}=\sigma_{0}^{2}+\sigma^{2} / n$. This is because $\bar{X} \sim N\left(\mu_{0}, \sigma_{\#}^{2}\right)$. We may also write $p_{\delta}(\lambda)=E e_{\delta, \lambda}(\mu)=\int_{-\infty}^{\infty} e_{\delta, \lambda}(\mu) f(\mu) d \mu$, where $e_{\delta, \lambda}(\mu)$ is the EPDL at (17).

6. In contrast to $P_{\mu}$ at (22), the unconditional probability of $\mu$ 's HPDR containing $\mu$ is exactly $95 \%$. This can be shown by integrating $P_{\mu} f(\mu)$ over the whole real line, but is most easily proved as follows:

$$
P(\mu \in H(\bar{X}))=E P(\mu \in H(\bar{X}) \mid \bar{X})=E(1-\alpha)=1-\alpha .
$$


7. In (25), $f(\bar{x}, s \mid \mu, \sigma)=f(\bar{x} \mid \mu, \sigma) f(s \mid \sigma)$,

where:

$$
\begin{aligned}
& f(\bar{x} \mid \mu, \sigma)=\phi((\bar{x}-\mu) \sqrt{n} / \sigma) \sqrt{n} / \sigma \\
& f(s \mid \sigma)=f(u \mid \sigma) d u / d s \\
& u=(n-1) s^{2} / \sigma^{2}, \quad d u / d s=2(n-1) s / \sigma^{2} \\
& f(u \mid \sigma)=u^{(1 / 2)(n-1)-1} e^{-u / 2} /\left\{2^{(1 / 2)(n-1)} \Gamma((n-1) / 2)\right\} .
\end{aligned}
$$

This follows because $\bar{X}$ and $S^{2}$ are conditionally independent, because $(\bar{X} \mid \mu, \sigma) \sim N\left(\mu, \sigma^{2} / n\right)$, and because $\left((n-1) S^{2} / \sigma^{2} \mid \mu, \sigma\right) \sim \chi^{2}(n-1)$.

8. Using numerical techniques to evaluate the integral at (25) exactly, we find that the PEPDL in Figure 11 equals 0.144 at $\lambda=0$ and has a maximum of 0.145 at $\lambda=0.9$.

9. Using numerical techniques to evaluate the integral at (25) exactly, we find that the PEPDL in Figure 12 equals 0.022 at $\lambda=0$. It also equals 0.043 at $\lambda=20$ and has a maximum of 0.043 at $\lambda=19.1$ (same to three decimals).

10. Suppose that the tail function is

$$
\tau(p)= \begin{cases}\delta_{1}, & 0 \leq p<\eta_{1} \\ \delta_{2}, & \eta_{1} \leq p<\eta_{2} \\ \delta_{3}, & \eta_{2} \leq p \leq 1\end{cases}
$$

where $0 \leq \eta_{1} \leq \eta_{2} \leq 1$ and $0 \leq \delta_{1} \leq \delta_{2} \leq \delta_{3} \leq 1$. Then the bounds of the associated $1-\alpha$ Clopper-Pearson $\mathrm{CI}$ are:

$$
\begin{aligned}
& l=\left\{\begin{array}{cc}
L\left(\alpha\left(1-\delta_{1}\right)\right) & \text { if } L\left(\alpha\left(1-\delta_{1}\right)\right) \leq \eta_{1} \\
\eta_{1} & \text { if } L\left(\alpha\left(1-\delta_{1}\right)\right)>\eta_{1} \text { and } L\left(\alpha\left(1-\delta_{2}\right)\right) \leq \eta_{1} \\
L\left(\alpha\left(1-\delta_{2}\right)\right) & \text { if } \eta_{1}<L\left(\alpha\left(1-\delta_{2}\right)\right) \leq \eta_{2} \\
\eta_{2} & \text { if } L\left(\alpha\left(1-\delta_{2}\right)\right)>\eta_{2} \text { and } L\left(\alpha\left(1-\delta_{3}\right)\right) \leq \eta_{2} \\
L\left(\alpha\left(1-\delta_{3}\right)\right) & \text { if } L\left(\alpha\left(1-\delta_{3}\right)\right)>\eta_{2}
\end{array}\right. \\
& u=\left\{\begin{array}{cc}
U\left(\alpha \delta_{3}\right) & \text { if } U\left(\alpha \delta_{3}\right) \geq \eta_{2} \\
\eta_{2} & \text { if } U\left(\alpha \delta_{3}\right)<\eta_{2} \text { and } U\left(\alpha \delta_{2}\right) \geq \eta_{2} \\
U\left(\alpha \delta_{2}\right) & \text { if } \eta_{1} \leq U\left(\alpha \delta_{2}\right)<\eta_{2} \\
\eta_{1} & \text { if } U\left(\alpha \delta_{2}\right)<\eta_{1} \text { and } U\left(\alpha \delta_{1}\right) \geq \eta_{1} \\
U\left(\alpha \delta_{1}\right) & \text { if } U\left(\alpha \delta_{1}\right)<\eta_{1}
\end{array}\right.
\end{aligned}
$$


11. This relationship also holds between the one-sided CI and all modified CP CI's defined by $\delta=\lambda=0$ and $0<\eta \leq 0.2589$. For $\eta>0.2589$ the modified $\mathrm{CI}$ is wider in expectation for values of $p$ in a neighbourhood of zero whose size increases as $\eta$ increases. The reason for this is that the one-sided CI at $x=0$ is $(0,0.2589)$, which follows from (34), whereby $u=1-0.05^{1 / 10}$ $=0.2589$. Thus any increase in $\eta$ past 0.2589 has the effect of making $u$ at (35) also increase to $\eta$ when $x=0$ (see Figure 19). The result is that the modified CI is wider than the one-sided CI when $x=0$ and so has a larger prior expected width if $p$ is sufficiently small.

12. For example, the Gaussian tail function (12) with $\delta=\lambda=0$ and $\eta=\bar{x}$ implies an automatic reduction in expected length (relative to the ordinary 95\% CI for $\mu$ in Section 4) of 16.1\% (the AGMPDL at (19) when $\alpha=0.05$ ). However, by (20) it also implies an actual coverage probability of only $90 \%$. 


\section{References}

Blaker, H. (2000). Confidence curves and improved exact confidence intervals for discrete distributions. The Canadian Journal of Statistics, 28, 783-798.

Blaker, H. (2001). Corrigenda: Confidence curves and improved exact confidence intervals for discrete distributions. The Canadian Journal of Statistics, 29, 681.

Blyth, C. R. and Still, H. A. (1983). Binomial confidence intervals. Journal of the American Statistical Association, 78, 108-116.

Brown, D. B., Cai, T. T. and DasGupta, A. (2001). Interval estimation for a binomial proportion. Statistical Science, 16, 101-133 (with discussion).

Casella, G. (1986). Refining binomial confidence intervals. The Canadian Journal of Statistics, 14, 113-129.

Clopper, C. J. and Pearson, E. S. (1934). The use of confidence or fiducial limits illustrated in the case of the binomial. Biometrika, 26, 404-413.

Lee, P.M., 1989. Bayesian Statistics: An Introduction. Oxford University Press, New York.

Puza, B. D, and O’Neill, T. J. (2004). Generalised Clopper-Pearson confidence intervals for the binomial proportion. Under review by the Journal of Statistical Computation and Simulation.

Stuart, A., Ord, J. K., and Arnold, S. (1999). Kendall's Advanced Theory of Statistics, Volume 2A. London: Arnold.

Venables, W. N., and Ripley, B. D. (1999). Modern Applied Statistics with S-Plus, 3rd ed. New York: Springer.

Venables, W. N., Smith, D. M., and the $R$ Development Core Team (2004). An Introduction to $R$. ISBN: 3-900051-05-4. Website: http://www.r-project.org

Wilson, E. B. (1927). Probable inference, the law of succession, and statistical inference. Journal of the American Statistical Association, 22, 209-212. 